\title{
Time-Varying Manual Control Identification in a Stall Recovery Task under Different Simulator Motion Conditions
}

\author{
Alexandru Popovici* \\ San Jose State University, NASA Ames Research Center \\ Peter M. T. Zaal ${ }^{\dagger}$ \\ San Jose State University, NASA Ames Research Center \\ Marc A. Pieters $\ddagger$ \\ San Jose State University, NASA Ames Research Center, and Delft University of Technology
}

\begin{abstract}
This paper adds data to help the development of simulator motion cueing guidelines for stall recovery training by identifying time-varying manual control behavior in a stall recovery task under different simulator motion conditions. A study was conducted with seventeen general aviation pilots in the NASA Ames Vertical Motion Simulator. Pilots had to follow a flight director through four stages of a high-altitude stall task. A timevarying identification method was used to quantify how pilot manual control parameters change throughout different stages of the task in both roll and pitch. Four motion configurations were used: no motion, generic hexapod motion, enhanced hexapod motion and full motion. Pilot performance was highest for the enhanced hexapod and full motion configurations in both roll and pitch, and the lowest without motion. In the roll axis, the pilot position gain did not significantly change throughout the stall task, but was the lowest for the condition with no motion. The pilot roll velocity gain was significantly different between motion conditions, the largest difference being found close to the stall point. The enhanced hexapod motion condition had the highest pilot roll velocity gain. In the pitch axis, the pilot position gain was significantly different between time segments but not between motion conditions. The pilot pitch velocity gain was highest for the full motion condition and increased close to the stall point, but did not change significantly for the other motion conditions. Overall, pilot control behavior under enhanced hexapod motion was most similar to that under full aircraft motion. This indicates that motion cueing for stall recovery training on hexapod simulators might be improved by using the principles behind the enhanced hexapod motion configuration.
\end{abstract}

\section{Nomenclature}

$A$

$e$

$f$

$H_{c} \quad$ controlled dynamics response

$H_{f} \quad$ motion filter response

$H_{o l} \quad$ open-loop response

$H_{p} \quad$ human controller response

$h \quad$ altitude, $\mathrm{ft}$

$I A S \quad$ indicated airspeed, kts

$K_{d} \quad$ aircraft dynamics gain, -

$K_{f} \quad$ motion filter gain, -

$K_{p} \quad$ pilot position gain, -

$K_{r t l}$ c.g. to p.s. translational acceleration gain, -

$\begin{array}{ll}K_{t r s} & \text { c.g. translational acceleration gain, - } \\ K_{v} & \text { pilot velocity gain, }- \\ k & \text { sinusoid index } \\ M R & \text { motion rating, \% } \\ N & \text { number of sine waves } \\ n & \text { pilot remnant } \\ n & \text { sinusoid frequency integer factor } \\ r & \text { reference attitude, deg } \\ S_{1-4} & \text { stall task segment } \\ s & \text { Laplace operator } \\ T & \text { thrust, lbf } \\ T_{L} & \text { pilot lead time constant, s } \\ t & \text { time, } \mathrm{s}\end{array}$

*Research Associate, Human Systems Integration Division, NASA Ames Research Center, Moffett Field, CA, 94035; alexandru.popovici@nasa.gov. Member.

†Senior Research Engineer, Human Systems Integration Division, NASA Ames Research Center, Moffett Field, CA, 94035; peter.m.t.zaal@nasa.gov. Member.

${ }^{\ddagger}$ Research Scholar, Human Systems Integration Division, NASA Ames Research Center, Moffett Field, CA, 94035; marc.a.pieters@ nasa.gov. 


$\begin{array}{llll}t_{1-4} & \text { stall segment end time, } \mathrm{s} & \text { Abbreviations } \\ u & \text { pilot control input, }- & \\ \alpha & \text { angle of attack, deg } & \text { DEKF } & \text { dual extended Kalman filter } \\ \delta_{a} & \text { aileron deflection, deg } & \text { EH } & \text { enhanced hexapod } \\ \delta_{e} & \text { elevator deflection, deg } & \text { FM } & \text { full motion } \\ \zeta_{f} & \text { motion filter damping ratio, }- & \text { GH } & \text { generic hexapod } \\ \zeta_{n} & \text { pilot neuromuscular damping ratio, }- & \text { GTM } & \text { General Transport Model } \\ \theta & \text { pitch angle, deg } & \text { MLE } & \text { maximum likelihood estimation } \\ \tau_{v} & \text { pilot time delay, s } & \text { NM } & \text { no motion } \\ \phi & \text { roll angle, deg } & \text { OMCT } & \text { Objective Motion Cueing Test } \\ \phi & \text { sinusoid phase, rad } & \text { PFD } & \text { primary flight display } \\ \varphi_{m} & \text { phase margin, deg } & \text { RMS } & \text { root mean square } \\ \omega_{c} & \text { crossover frequency, rad s } & \text { VAF } & \text { variance accounted for } \\ \omega_{d} & \text { aircraft dynamics break frequency, rad s } & \text { VMS } & \text { Vertical Motion Simulator } \\ \omega_{f} & \text { motion filter break frequency, rad s }{ }^{-1} & & \\ \omega_{n} & \text { pilot neuromuscular frequency, } \operatorname{rad~s}^{-1} & & \end{array}$

\section{Introduction}

This paper identifies time-varying manual control behavior in a stall recovery task under different simulator motion conditions to help develop motion cueing guidelines for stall recovery training. Today, airline pilots only receive training in recognizing and recovering from an approach to stall, but not in full stall recovery. Starting in 2019, airline pilots will be required to perform full stall recovery training in flight simulators. ${ }^{1}$ However, most training simulators are not set up to provide this training currently, as they do not accurately represent aircraft behavior in upset situations that take the aircraft out of its normal flight envelope. ${ }^{2}$ Post-stall aircraft models need to be implemented to correctly simulate the aircraft response after the stall point. In addition, motion cues need to adequately represent this response to ensure the skills learned in simulator training are directly usable in real flight.

Many previous studies investigated the effects of motion cues on manual control skills in different tasks and environments. $^{3-8}$ These studies mostly focused on the identification of time-invariant control behavior in single-axis tasks with constant task variables, a limitation largely imposed by the human control behavior identification techniques available at the time. However, under the highly variable conditions in a stall recovery maneuver, pilot manual control behavior is expected to vary significantly due to changes in control effectiveness and aircraft dynamic behavior. The direct applicability of previous pilot behavioral observations in motion flight simulation studies to this scenario might not be straightforward. In the last decades, some progress has been made to identify control behavior under timevarying conditions or in real flight tasks. ${ }^{9-12}$ However, the developed approaches either do not allow for a direct estimation of pilot model parameters from time-domain signals, or require a priori assumptions about the changes in manual control parameters. Recently, a new parameter estimation technique based on a dual extended Kalman filter (DEKF) was developed that allows for the direct estimation of pilot model parameters in time-varying tasks such as a stall recovery. ${ }^{13}$

This paper adds data to the current literature as follows. It is the first study to utilize a novel pilot modeling and identification technique based on a DEKF that allows for the direct estimation of pilot control behavior parameters in time-varying tasks such as a stall recovery. Second, using this technique, pilots' adaptation to different simulator motion settings in different segments of a stall recovery maneuver was investigated. Third, this study used the NASA Vertical Motion Simulator (VMS), which allows for inclusion of a full motion condition that represents real aircraft motion. Last, a sufficiently large pilot pool added statistical reliability to the results.

The paper is structured as follows. The stall recovery task is described in Section II, after which the experiment setup is discussed in Section III. The results are provided in Section IV, followed by a discussion and conclusions in Sections V and VI, respectively.

\section{Control Task}

Pilots performed a high-altitude stall recovery task while compensating for disturbances in both the roll and pitch degrees of freedom simultaneously. A flight director on a primary flight display (PFD) guided the pilots through the stall maneuver. A block diagram of the control task is depicted in Fig. 1.

Aircraft roll and pitch attitudes $\phi$ and $\theta$ were subtracted from the desired roll and pitch attitudes $r_{\phi}$ and $r_{\theta}$ to 


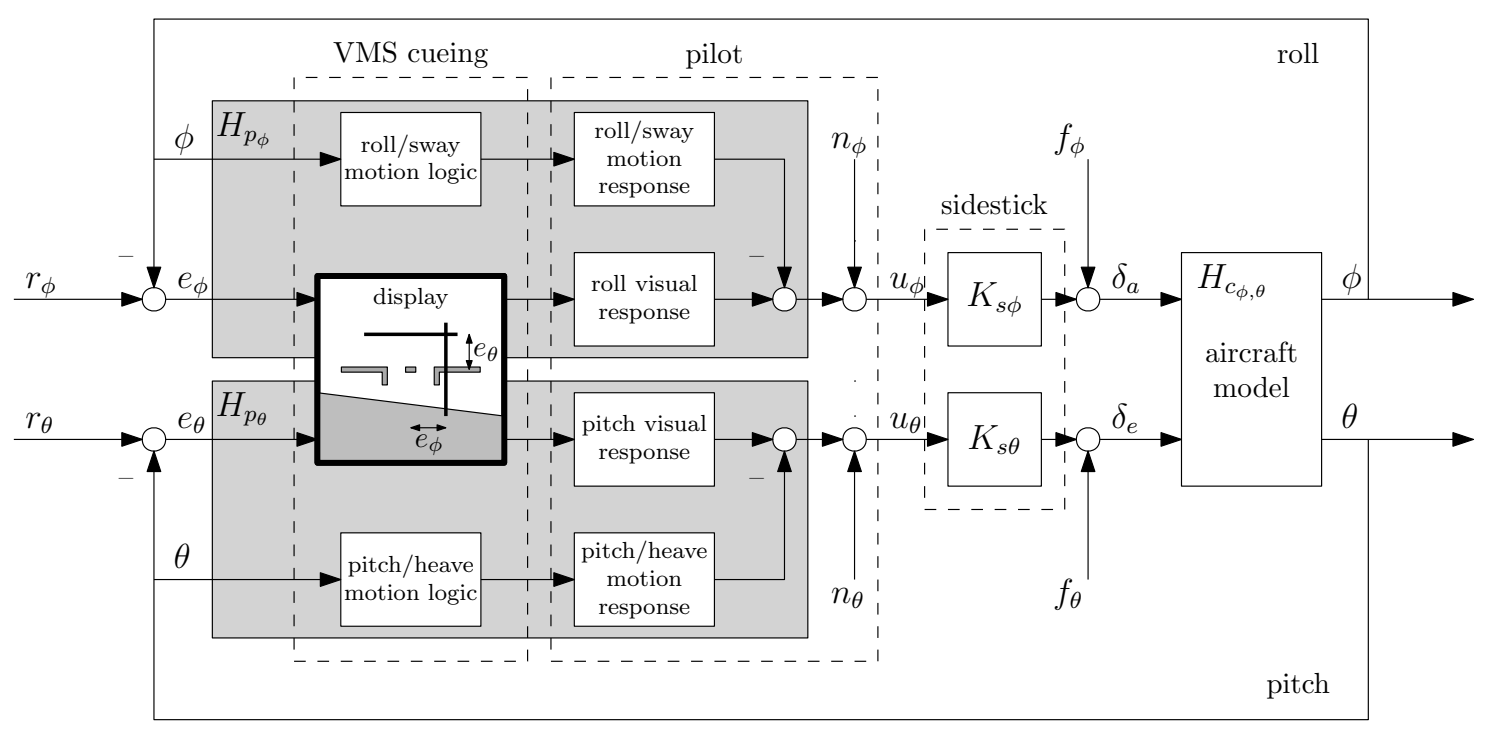

Figure 1. Closed-loop control task.

create roll and pitch errors $e_{\phi}$ and $e_{\theta}$ which were indicated by the flight director bars on the PFD. It was pilots' task to minimize the aircraft roll and pitch errors by making control inputs $u_{\phi}$ and $u_{\theta}$ using a sidestick with gains $K_{s \phi}$ and $K_{s \theta}$. To allow for the identification of pilot control behavior, two independent disturbance forcing functions $f_{d \phi}$ and $f_{d \theta}$ were used in the roll and pitch axes, respectively, that were a summation of ten sine waves (Section D). The forcing functions induced disturbances similar to atmospheric turbulence. Pilots performed the stall task in the clouds; that is, the out-the-window view did not have any visual features that could be used to determine the attitude of the aircraft.

The stall task was divided into four segments as shown in Fig. 2. In the first segment from 0 to $82 \mathrm{~s}(S 1)$, task parameters remained at the initial trim condition. This segment was used to compare pilot control behavior identified using the newly developed DEKF parameter estimation technique to that identified using traditional methods for timeinvariant control behavior. During the second segment from 82 to $124 \mathrm{~s}(S 2)$, pilots flew the aircraft into a stall by following an increased reference pitch attitude. Thrust was set to idle automatically during this segment to get to the stall point faster. The reference pitch attitude dropped below zero in the third segment from 124 to $152 \mathrm{~s}$ (S3). By following this nose-down reference pitch attitude, the angle of attack was reduced to recover from the stall. During this segment, maximum thrust was applied automatically to regain airspeed faster. After a slow increase of the reference pitch such that secondary stalls did not occur, pilots controlled the aircraft for another 30 seconds around the original pitch trim attitude in the fourth segment $(S 4)$. The desired roll attitude remained zero during all four segments of the stall recovery task; that is, pilots had to keep the wings level at all times. Segments $S 2$ to $S 4$ had a transition phase at the beginning in which the reference pitch attitude changed gradually to the new reference value. Data from these transitions were not used in the analysis.

Example recordings for attitude, angle of attack, altitude, airspeed, and thrust during the task are depicted in Fig. 3. The reference signals for roll and pitch are also provided in the top two plots. The transition phases are the areas in between the dashed vertical lines in each plot. Fig. 3 shows that the altitude and airspeed remained approximately constant during $S 1$. In $S 2$, altitude increased, indicated airspeed decreased, and angle of attack increased gradually up to the critical angle of attack around $16 \mathrm{deg}$. During the recovery in $S 3$, the angle of attack rapidly decreased, altitude decreased and airspeed increased. Finally, in the last segment, the reference pitch attitude had the same value as in the first segment. Angle of attack remained approximately constant, altitude increased, and airspeed decreased slightly.

\section{A. Aircraft Model}

The General Transport Model (GTM) was used to simulate the aircraft dynamics. This full-scale simulation model is representative of a generic aircraft similar to a Boeing 757, and includes accurate post-stall dynamics. ${ }^{14-16}$ This model was developed from a sub-scale polynomial aerodynamic database, extended to cover the stall regime with windtunnel and spin-tunnel test data. The model was further adapted to represent a full-scale aircraft by making Reynolds Number corrections. The model used a basic yaw damper. All other stability and control augmentation functions were 


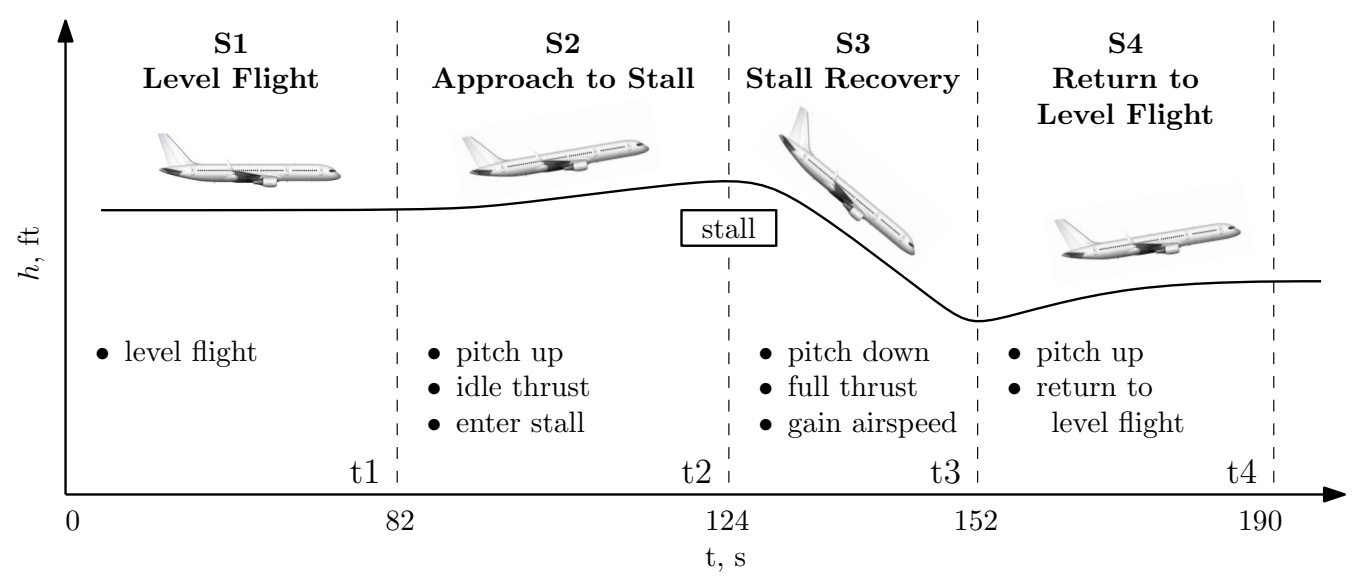

Figure 2. Stall task segments.
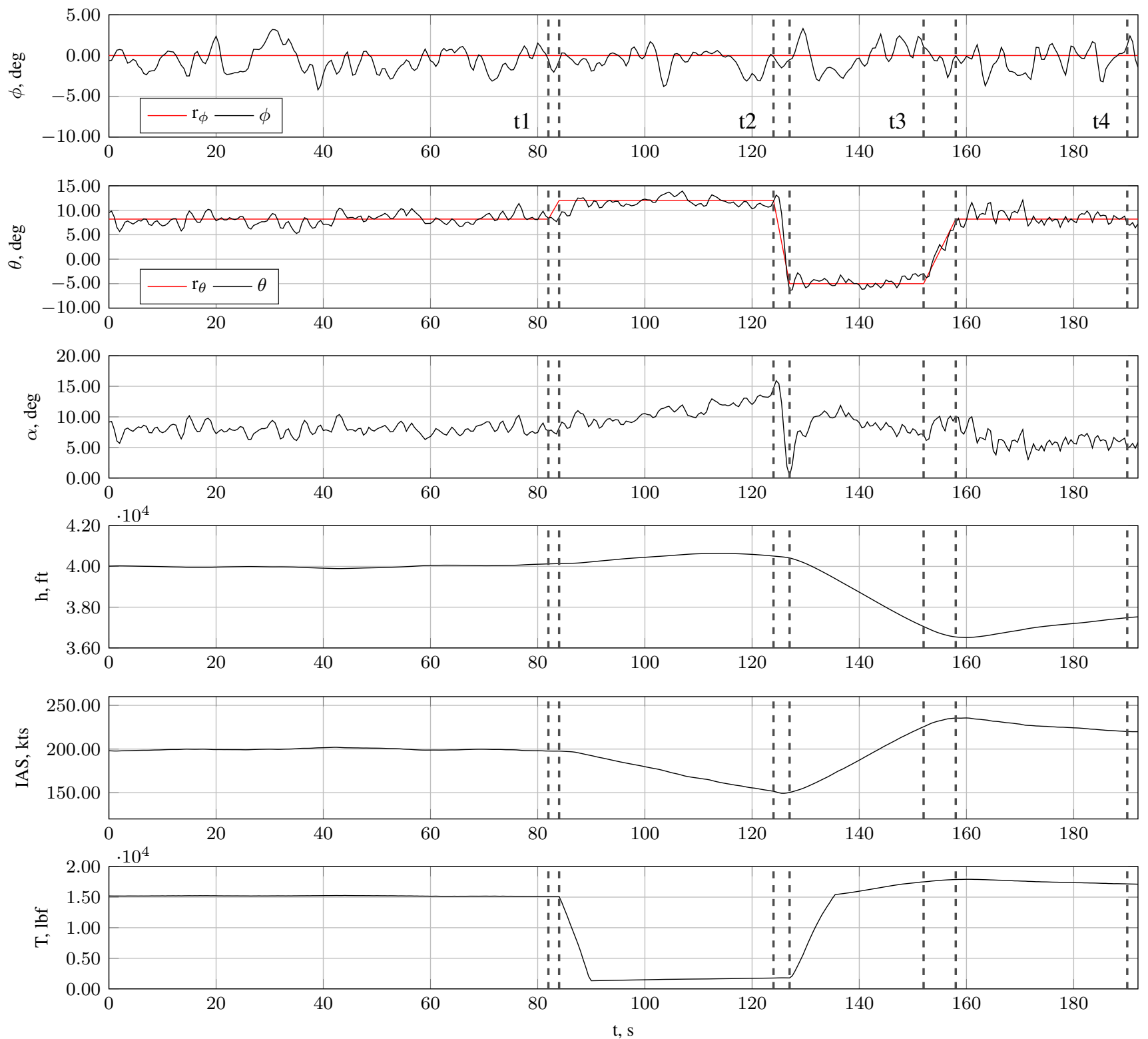

Figure 3. Flight variable recordings. 
switched off. The simulation model was implemented in the FLTz simulation environment. ${ }^{17}$ At the start of each run the aircraft was trimmed at an altitude of $40,000 \mathrm{ft}$ and a true airspeed of $382 \mathrm{kts}$. The gross weight of the airplane was $185,000 \mathrm{lbs}$.

\section{B. Pilot Model}

From a control-theoretic perspective, the visual and motion cues in roll and pitch were the inputs to the pilot model and the control inputs were the outputs (Fig. 1). This warrants the identification of a pilot visual and motion response channel in both degrees of freedom. However, the DEKF parameter estimation technique developed in Ref. 13 currently only allows for the identification of a single channel. This means only a lumped response function, combining the visual and motion responses, could be identified. For different reasons, this approach was also taken in previous research investigating the effects of motion cues on pilot control behavior. ${ }^{18}$ Cross coupling in pilot control behavior between roll and pitch was not modeled explicitly.

Using the recorded error and control input signals in roll and pitch, the parameters of a pilot model with response functions $H_{p_{\phi}}$ and $H_{p_{\theta}}$ were identified using the DEKF parameter estimation technique. The differences between the pilot model response function outputs and the measured pilot control inputs were the remnants $\left(n_{\phi}\right.$ and $\left.n_{\theta}\right)$, which account for nonlinear behavior and noise. Note that visual display and simulator motion dynamics are also captured by the pilot response functions.

A human operator adapts his or her control behavior to the controlled dynamics such that the combined human operator controlled dynamics open-loop response approximates a single integrator in the region of the crossover frequency, according to the crossover model theorem. ${ }^{19}$ Because of the typical characteristics of the transport aircraft dynamics used, the human operator was required to generate lead, especially closer to the stall point when the dynamics become more unstable. Hence, the pilot transfer functions in both roll and pitch were defined by:

$$
H_{p_{\phi}}(s, t)=H_{p_{\theta}}(s, t)=\left(K_{p}(t)+K_{v}(t) s\right) e^{-\tau_{v} s} \frac{\omega_{n}^{2}(t)}{s^{2}+2 \zeta_{n}(t) \omega_{n}(t) s+\omega_{n}^{2}(t)}
$$

in which the pilot equalization dynamics are characterized by the pilot position gain $K_{p}$ and the pilot rate gain $K_{v}$, or the more commonly used lead time constant $T_{L}$. In Eq. (1), $K_{v}=K_{p} T_{L}$. Note that $1 / T_{L}$ defines the frequency where lead starts to be generated. Human controller limitations are captured by the time delay $\tau_{v}$, neuromuscular frequency $\omega_{n m}$, and neuromuscular damping $\zeta_{n m}$. In order to estimate these parameters using the DEKF technique, the transfer function in Eq. (1) had to be converted to a state-space representation using a 3rd order Padé approximation for the time delay. ${ }^{13}$ Furthermore, the time delay was kept constant to minimize the possibility of filter divergence in case not enough control input was provided.

\section{Open-Loop Dynamics}

Using the control diagram in Fig. 1, the open-loop responses for both roll and pitch were defined by:

$$
H_{o l_{\phi, \theta}}(s, t)=H_{p_{\phi, \theta}}(s, t) K_{s_{\phi, \theta}} H_{c_{\phi, \theta}}(s, t)
$$

The crossover frequency $\left(\omega_{c_{\phi, \theta}}\right)$ is the frequency where the magnitude of the open-loop response is 1.0. At this crossover frequency, the phase difference from -180 degrees is the phase margin $\left(\varphi_{m_{\phi, \theta}}\right)$.

A frequency response of the aircraft dynamics was necessary to compute the open-loop characteristics of the pilot-aircraft system using Eq. (2). The approach was to use a highly simplified form of the aircraft dynamics:

$$
H_{d}(s, t)=\frac{K_{d}(t)}{s\left(s+\omega_{d}(t)\right)}
$$

where $K_{d}$ is the aircraft dynamics gain and $\omega_{d}$ the break frequency where the aircraft response transitions from a single integrator to a double integrator. Both the gain and break frequency were time variant and estimated using an extended Kalman filter technique.

\section{Forcing Functions}

The pitch and roll forcing functions were sum-of-sines signals defined by Eq. (4), with $A(k), \omega(k)$ and $\phi(k)$ the amplitude, frequency and phase of the $k^{t h}$ sine in $f_{\theta}$ or $f_{\phi}$, respectively. $N=10$ represents the number of sine waves. 
Table 1. Forcing function properties.

\begin{tabular}{|c|c|c|c|c|c|c|c|c|}
\hline \multirow[b]{2}{*}{$\begin{array}{l}k \\
-\end{array}$} & \multicolumn{4}{|c|}{ Pitch Disturbance, $f_{\theta}$} & \multicolumn{4}{|c|}{ Roll Disturbance, $f_{\phi}$} \\
\hline & $\begin{array}{c}n_{\phi} \\
-\end{array}$ & $\begin{array}{c}\omega_{\phi} \\
\operatorname{rad~s}{ }^{-1}\end{array}$ & $\begin{array}{l}A_{\phi} \\
\text { deg }\end{array}$ & $\begin{array}{l}\phi_{\phi} \\
\mathrm{rad}\end{array}$ & $\begin{array}{c}n_{\theta} \\
-\end{array}$ & $\begin{array}{c}\omega_{\theta} \\
\operatorname{rad} s^{-1}\end{array}$ & $\begin{array}{l}A_{\theta} \\
\text { deg }\end{array}$ & $\begin{array}{l}\phi_{\theta} \\
\mathrm{rad}\end{array}$ \\
\hline 1 & 3 & 0.230 & 0.0030 & 0.9164 & 2 & 0.153 & 0.0030 & 1.7664 \\
\hline 2 & 7 & 0.537 & 0.0030 & 2.0647 & 5 & 0.384 & 0.0030 & 0.8532 \\
\hline 3 & 13 & 0.997 & 0.0030 & 1.2614 & 11 & 0.844 & 0.0030 & -2.2495 \\
\hline 4 & 27 & 2.071 & 0.0030 & 1.5388 & 23 & 1.764 & 0.0030 & -1.0488 \\
\hline 5 & 41 & 3.145 & 0.0036 & 0.6213 & 37 & 2.838 & 0.0037 & 1.9860 \\
\hline 6 & 53 & 4.065 & 0.0040 & 0.7320 & 51 & 3.912 & 0.0043 & -1.0641 \\
\hline 7 & 73 & 5.599 & 0.0046 & -2.6738 & 71 & 5.446 & 0.0050 & 1.8933 \\
\hline 8 & 103 & 7.900 & 0.0058 & -1.5389 & 101 & 7.747 & 0.0064 & 1.9704 \\
\hline 9 & 139 & 10.661 & 0.0077 & -2.7371 & 137 & 10.508 & 0.0085 & -0.0342 \\
\hline 10 & 174 & 13.346 & 0.0101 & -2.1734 & 171 & 13.116 & 0.0110 & -2.1796 \\
\hline
\end{tabular}

$$
f(t)=\sum_{k=1}^{N} A(k) \sin [\omega(k) t+\phi(k)]
$$

Two disturbance forcing functions were present, one for roll and one for pitch. A summary of all forcing function parameters can be found in Table 1.

The sinusoid frequencies were all integer multiples of the measurement time base frequency, $\omega=0.0767 \mathrm{rad} / \mathrm{s}$. The selected integer multiples were used in earlier experiments. They were chosen to fully cover the range of human control at regular intervals on a logarithmic scale. ${ }^{20}$

The amplitudes of the sinusoids were determined using a second-order low-pass filter. The amplitudes of the first four sine waves were manually changed in order to keep the aircraft elevator and aileron deflections within their maximum deflections and the motion of the simulator within the boundaries of the motion envelope throughout the stall task. The final amplitude distribution of the pitch disturbance signal was scaled to obtain a variance of $0.4 \mathrm{deg}^{2}$. For roll the variance was higher, $5.0 \mathrm{deg}^{2}$, since it was found that the roll error was more difficult to perceive on the PFD. ${ }^{20}$ To determine the phase distributions, a high number of phase sets was randomly generated. Two sets were selected that created signals with a Gaussian-like distribution and an average crest factor. The resulting disturbance-rejection task was challenging, but not overly difficult.

\section{E. Simulator Motion}

The experiment used four motion configurations. The standard VMS motion algorithm and hardware were used for all four motion configurations. The equivalent time delays of the VMS motion system for the pitch, roll, yaw, longitudinal, lateral and vertical axes are 47, 68, 48, 50,69 and $67 \mathrm{~ms}$, respectively. More details about the motion algorithm are provided in Ref. 21. The VMS motion logic consists of second-order high-pass filters to attenuate the rotational and translational aircraft model accelerations:

$$
H_{f}(s)=K_{f} \frac{s^{2}}{s^{2}+2 \zeta_{f} \omega_{f} s+\omega_{f}^{2}}
$$

where $K_{f}$ is the motion gain, and $\zeta_{f}$ and $\omega_{f}$ are the motion filter damping ratio and break frequency, respectively.

The four motion configurations not only differed in motion filter parameter settings, but also in the type of translational accelerations simulated. Translational accelerations at the pilot station are a combination of translational accelerations of the aircraft's center of gravity (c.g.) and translational accelerations as a result of the pilot station (p.s.) rotating with respect to the center of gravity (Fig. 4). Gains on each of the translational acceleration components $\left(K_{t r s}\right.$ and $K_{r t l}$ ) allowed for a different weighting of each component.

The four motion conditions were similar to the ones in Ref. 20. In the no-motion condition $(N M)$, the simulator was operating with the motion system engaged; however, with all motion parameters set to zero. In the hexapod motion configurations, the motion was attenuated to fit within the motion space of a typical 60-inch, six-legged hexapod simulator. The generic hexapod condition $(G H)$ provided motion cuing based on the current industry standard. The motion response of the VMS for this condition was matched to the average response of a statistical sample of eight 


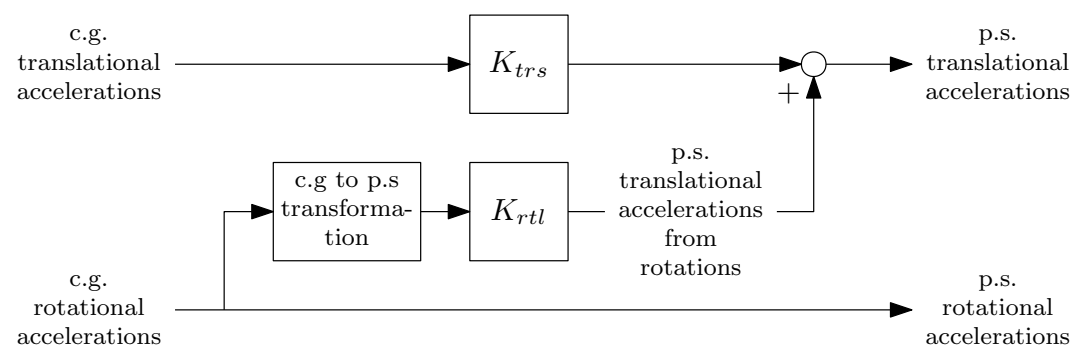

Figure 4. Transformation from center of gravity to pilot station accelerations.

Table 2. Motion logic parameters.

\begin{tabular}{cccc}
\hline \hline \multicolumn{4}{c}{ Motion Component Gains } \\
\hline & GH & EH & FM \\
\hline$K_{\text {trs }}$ & 1.00 & 0.00 & 1.00 \\
$K_{\text {rtl }}$ & 1.00 & 0.75 & 1.00
\end{tabular}

\begin{tabular}{|c|c|c|c|}
\hline \multicolumn{4}{|c|}{ Washout Gains } \\
\hline & GH & EH & FM \\
\hline$K_{f x}$ & 0.58 & 0.50 & 0.58 \\
\hline$K_{f y}$ & 0.72 & 1.00 & 1.00 \\
\hline$K_{f z}$ & 0.63 & 0.80 & 0.80 \\
\hline$K_{f p}$ & 0.74 & 1.00 & 1.00 \\
\hline$K_{f q}$ & 0.86 & 0.90 & 0.90 \\
\hline$K_{f r}$ & 0.63 & 1.00 & 1.00 \\
\hline
\end{tabular}

\begin{tabular}{cccc}
\hline \multicolumn{4}{c}{ Washout Break Frequencies } \\
\hline & GH & EH & FM \\
\hline$\omega_{f x}$ & 2.04 & 0.80 & 0.80 \\
$\omega_{f y}$ & 0.87 & 0.20 & 0.20 \\
$\omega_{f z}$ & 2.76 & 1.00 & 1.00 \\
$\omega_{f p}$ & 1.06 & 0.20 & 0.20 \\
$\omega_{f q}$ & 0.49 & 0.25 & 0.25 \\
$\omega_{f r}$ & 0.77 & 0.20 & 0.20 \\
\hline \hline
\end{tabular}

\begin{tabular}{cccc}
\hline \hline \multicolumn{4}{c}{ Washout Damping Ratios } \\
\hline & GH & EH & FM \\
\hline$\zeta_{f x}$ & 0.58 & 0.71 & 0.71 \\
$\zeta_{f y}$ & 1.57 & 0.71 & 0.71 \\
$\zeta_{f z}$ & 1.10 & 0.71 & 0.71 \\
$\zeta_{f p}$ & 1.18 & 0.71 & 0.71 \\
$\zeta_{f q}$ & 1.22 & 0.71 & 0.71 \\
$\zeta_{f r}$ & 1.10 & 0.71 & 0.71 \\
\hline \hline
\end{tabular}

simulators using the Objective Motion Cueing Test (OMCT) ${ }^{22}$ Both the translational acceleration components from the c.g. and as a result of rotations with respect to the c.g. were simulated in this condition.

The enhanced hexapod motion condition $(E H)$ provided motion optimized for stall recovery training based on the findings of our previous experiments. This motion condition only simulated the translational accelerations as a result of rotations with respect to the c.g. Taking out the c.g. component of the total pilot-station translational accelerations resulted in a condition where hardly any attenuation of the remaining accelerations was required, leading to washout gains of 1.00 and break frequencies of 0.20 for most degrees of freedom. The full VMS motion condition $(F M)$ used the entire VMS motion envelope to simulate motion with the highest possible fidelity. In this motion condition both translational acceleration components were simulated with the motion logic parameter settings of condition $E H$; that is, with minimal attenuation.

Parameter settings for all motion configurations are provided in Table 2. Details on the tilt coordination were omitted from this discussion for brevity; however, tilt coordination was present in each motion configuration. Although an important cue for pilots to recognize an impending stall, buffet motion was not simulated in this experiment, as the main focus was to model the pilot's response to motion cues reflecting the changes in aircraft dynamics during a stall maneuver.

\section{Experiment Setup}

\section{A. Method}

\section{Independent Variable}

The experiment had a within-subjects design with one independent variable. The independent variable was the motion configuration with four levels: no motion (NM), generic hexapod motion $(\mathrm{GH})$, enhanced hexapod motion (EH), and full VMS motion (FM). The motion configurations are detailed in Section II.E.

\section{Apparatus}

The experiment was conducted in the VMS with the transport aircraft cab (T-cab), see Fig. 5. This cab has two seats. The left seat had a wheel and column to make control inputs. Participants performed the experiment from the right seat, which had a sidestick on the right side to make control inputs. Rudder pedals were available for both seats, however these were not operational during the experiment. Throttle levers were located in between the seats. The throttles were in auto-throttle mode and were automatically backdriven without pilot inputs. A PFD with representative V-speed 


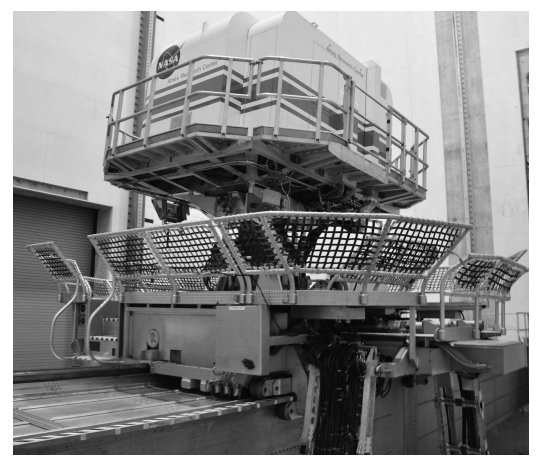

Figure 5. Vertical Motion Simulator.

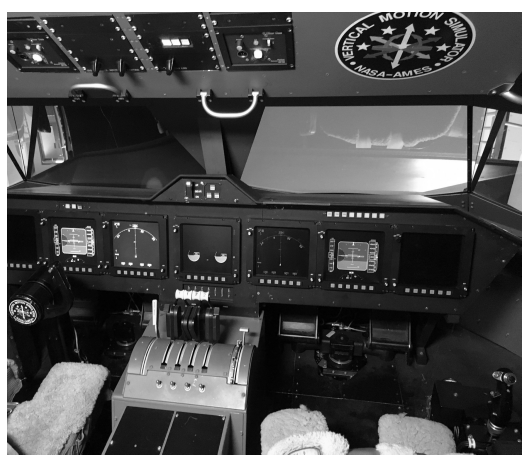

Figure 6. Cockpit setup.

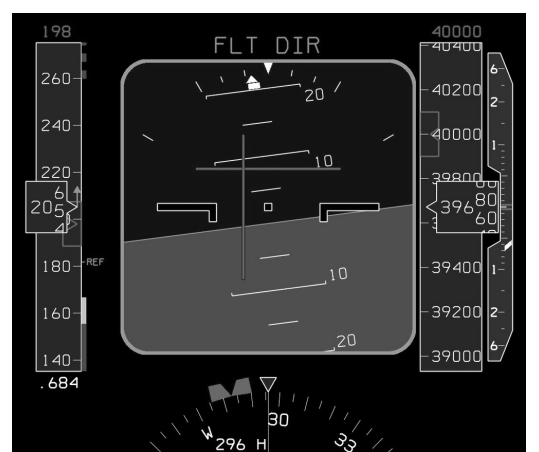

Figure 7. Primary flight display.

markings, a navigation display, and an engine display were located in front of both seats (Fig. 6).

The out-the-window visual cues of T-CAB were collimated and provided by a system that projected a high-quality image on six spherical mirrors. The mirrors formed a dome-like section providing a continuous field-of-view image to both pilots. The out-the-window visual had a $220^{\circ}$ horizontal field of view and a $28^{\circ}$ vertical field of view $\left(10^{\circ}\right.$ up and $18^{\circ}$ down). A Rockwell-Collins EPX5000 computer image generator created the out-the-window visual scene. The visual system equivalent time delay was $62 \mathrm{~ms} .^{21}$ This was in line with the equivalent time delays of the motion system (Section E). The out-the-window visual system provided a visual scene in the clouds, without visual features that could be used to determine the attitude of the aircraft.

\section{Procedures}

Before the start of the experiment, pilots received an extensive briefing explaining the main purpose of the experiment and the general procedures. Pilots were told the motion settings changed between runs; however, no specifics were given about the different motion conditions. After the briefing, pilots filled out a short questionnaire with questions about their aircraft and simulator experience, after which they signed an informed consent form. After a safety walkaround and an explanation of the relevant simulator cab features, pilots were provided with an example run of the task by the experimenter showing how to compensate for the disturbances more effectively. Pilots were instructed to give smooth and continuous control inputs. To motivate pilots to perform better, they were told the current best performance score (Section 5) and encouraged to improve on it.

Pilots performed eight runs per motion condition (including training runs) for a total of 32 runs. The four motion conditions were presented in random order according to a balanced Latin square design. Each run lasted 190 seconds. The total run time of the experiment was three and a half hours, including briefing and break time. Two or three runs for each condition were performed in between 15-minute breaks. Participants were allowed to take longer brakes if requested. After completing all measurement runs, the final four runs (one run for each motion configuration) were used to gather subjective motion ratings. Pilots were told they could control more freely during these four runs to get a better feel of the motion whilst still performing the stall task. The first run of the last four always used the full motion configuration. Pilots were told this was the baseline full-motion condition. The three remaining motion conditions were then presented randomly and pilots were asked to rate the motion of these conditions against the baseline by providing a percentage between 0 and $100 \%$ at the end of each run. They were also asked to provide general comments about the motion.

\section{Participants}

Seventeen general aviation pilots participated in the experiment. The most experienced pilot had 2,400 flight hours. The average number of flight hours over all participants was 373 hours, with a standard deviation of \pm 582 hours. Furthermore, pilots had an average of 21 hours of flight simulator experience, with a standard deviation of \pm 27 hours. Pilots had flown an average of 48 hours in the past three months, with a standard deviation of \pm 51 hours, both in the simulator and in actual flight. The youngest pilot was 19 years old and the oldest was 38 . The average age was 26.9 years, with a standard deviation of \pm 6.1 years. All participants were comfortable with operating the joystick with their right hand. Pilots were compensated for their participation. 


\section{Dependent Measures}

The dependent measures considered in this experiment were motion rating, task performance and control activity, pilot model parameters and pilot-aircraft open-loop characteristics. The motion ratings scored the motion fidelity of the $N M, G H, E H$ conditions against the $F M$ condition, in percentages from 0 (not similar to $F M$ ) to $100 \%$ (equivalent to $F M$ ). The full motion condition had a default rating of $100 \%$.

Pilot performance and control behavior parameters were a function of time. To allow for a comparison of these dependent measures between key points in the stall maneuver, the mean values over the last two seconds of each segment of the stall task $(S 1-S 4)$ were determined. These time segments are indicated by $t 1$ to $t 4$ in Fig. 2 . Pilot performance and control activity were defined by the root mean square of the attitude errors $\left(R M S_{e}\right)$ and control inputs $\left(R M S_{u}\right)$, respectively, for both roll and pitch, for all four time segments of the stall task.

Pilot model parameters were identified using attitude errors and control inputs in roll and pitch averaged over four runs of each motion condition. A DEKF was used to identify pilot position and velocity gains $\left(K_{p}, K_{v}\right)$, neuromuscular parameters $\left(\omega_{n}, \zeta_{n}\right)$ and a time delay $\left(\tau_{v}\right) .{ }^{13}$ Equalization and neuromuscular parameters were considered time-varying during the run, whereas the time delay was kept constant to minimize the possibility of filter divergence in case not enough control input was provided. Initial parameter estimates were obtained with a maximum likelihood estimation (MLE) method using the first 82 seconds of level flight of the averaged data for each motion condition. ${ }^{23}$

The parameters $K_{d}$ and $\omega_{d}$ of the aircraft dynamics approximation in Eq. (3) were estimated using an extended Kalman filter. A MLE method was also used in this case to obtain initial parameter estimates from the constant level flight of $82 \mathrm{~s}$ in $S 1$ to initialize the extended Kalman filter. In this filter, the aircraft parameters were included in the augmented state and estimated for the entire stall recovery maneuver. Finally, the open-loop characteristics were determined using Eq. (2) in order to determine the crossover frequency and phase margin for each motion condition and time segment.

\section{B. Hypotheses}

This study used motion configurations similar to those in a previous experiment. ${ }^{20}$ The generic hexapod motion condition $(G H)$ had motion similar to what current hexapod training simulators provide. The enhanced hexapod motion condition $(E H)$ eliminated translational c.g. accelerations to allow for increased fidelity of the translational accelerations as a result from rotations about the c.g. and the rotational accelerations themselves; that is, $E H$ had a higher fidelity of the motion cues most important for aircraft control during the stall task compared to $G H$. For tracking tasks with controlled elements requiring lead equalization, such as the aircraft dynamics used in this study, motion feedback is used by human controllers to reduce the amount of visually generated lead, allowing for better disturbance-rejection performance. ${ }^{24}$ The extent to which motion feedback is used is affected by the fidelity of motion stimuli important to the task. Attenuation of these motion cues, either by scaling or high-pass filtering, results in human manual control with lower gains and increased reliance on visual lead, which typically results in worse disturbancerejection performance. As the stability of the aircraft dynamics decreases closer to the stall point, motion becomes more important to maintain a certain level of performance. A new pilot control behavior identification technique based on a DEKF was used for the first time to investigate how pilot model parameters vary during a stall maneuver under the different motion configurations. Based on these considerations, the literature, and test runs in the VMS, the following hypotheses were formulated:

H1: Performance of the DEKF - The DEKF was expected to provide more accurate and consistent results when identifying pilot control behavior in roll compared to pitch as it was easier to perceive and act on roll disturbances. In addition, pitch control inputs had to be adjusted for the DEKF procedure as the measurements were not centered around zero.

H2: General Effects of Motion on Control Behavior - Pilot control behavior in the $E H$ motion condition was expected to be more similar to the $F M$ condition compared to $G H$. Pilot velocity gains were expected to be higher under the motion configurations with higher motion gains and lower break-frequencies ( $E H$ and $F M)$, leading to higher open-loop crossover frequencies and lower phase margins. It was anticipated pilots would have the smallest disturbance-rejection error $\left(R M S_{e}\right)$ in the $E H$ and $F M$ conditions and the largest in the $N M$ condition.

H3: Pilot Adaptations to Changing Dynamics - It was anticipated that pilots' control behavior and use of motion cues would adapt to the changing aircraft dynamics and conditions throughout the stall task. As the aircraft dynamics become more unstable close to the stall point $(t 2)$, the pilot velocity gain was expected to increase 
while the pilot position gain was not expected to change, resulting in a higher emphasis on visually generated lead.

H4: Differences Between Motion Configurations over Time - It was hypothesized that differences in pilot control behavior and performance between the different motion configurations would increase closer to the stall point as motion cues become more important to maintain the same level of disturbance-rejection performance.

\section{Results}

This section presents the combined results of 17 pilots that participated in the experiment. Error bar plots depict means and 95\% confidence intervals over all pilots for each motion condition and time segment after the data was corrected for between-subject variability. For time-varying dependent variables, means and confidence intervals were calculated from data of the last 2 seconds of each stall segment ( $S 1, S 2, S 3$ and $S 4$ ), see Fig. 2.

A two-way repeated measures analysis of variance (ANOVA) was performed to detect statistically significant interactions between motion configuration and time segment of the stall recovery maneuver (motion $\times$ time) for each dependent measure, or the simple main effects of motion or time. The four levels of motion were: no motion $(N M)$, generic hexapod $(G H)$, enhanced hexapod $(E H)$, and full motion $(F M)$. The four time intervals during the stall recovery maneuver were: level flight $(t 1)$, approach to stall $(t 2)$, dive ( $t 3)$, and recovery ( $t 4)$. Pilot time delays for both pitch and roll were considered constant and motion ratings were considered for the entire stall task only. Therefore, a one-way ANOVA was performed for these variables to investigate significant differences between motion conditions only. An overview of the main statistical test results is given in Table 3.

Data from certain pilots were removed due to inaccurate parameter estimates. No outliers were present, as no studentized residuals were outside \pm 3 standard deviations from the mean for each dependent variable.

\section{A. Motion Ratings}

In Fig. 8, pilot subjective motion ratings $(M R)$ are presented. A one-way repeated measures ANOVA was performed to investigate the effect of motion condition on subjective motion perception. The FM condition was rated at $100 \%$ by default, and the other motion conditions had to be compared relative to this condition. There was a statistically significant effect of motion $(p<0.001)$, see Table 3. Post-hoc analysis with a Bonferroni adjustment showed that $M R$ increased from $0.6 \%$ for $N M$ to $66.6 \%$ for $G H$ ( $p<0.001)$, to $80.4 \%$ in $E H$ $(p<0.001)$, and to $100.0 \%$ for $F M(p<0.001)$. There was no significant difference in motion ratings between $G H$ and $E H(p=0.082)$. $M R$ significantly increased from 66.6 in $G H$ to 100.0 in $F M(p<0.001)$. Lastly, motion ratings significantly increased from 80.4 in $G H$ to 100.0 in $F M$.

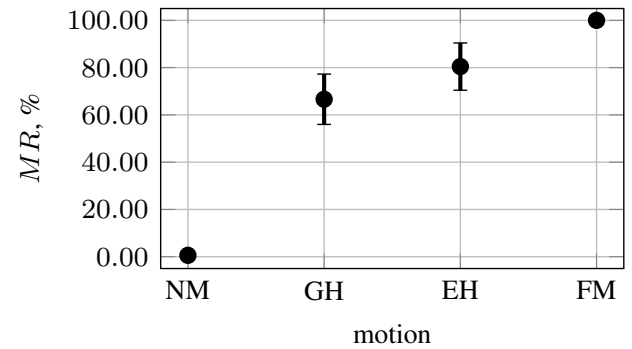

Figure 8. Motion ratings.

\section{B. Pilot Performance and Control Activity}

Roll error and control input RMS are presented in Fig. 9. A lower $R M S_{e}$ and $R M S_{u}$ indicate better performance and lower control activity, respectively. In Fig. 9a, no statistically significant two-way interaction between motion and time was found for the RMS of the roll error signal. The main effect of motion introduced significant differences in $R M S_{e}$ between conditions $(p=0.001)$. Post-hoc analysis with a Bonferroni adjustment indicated a significant decrease from $1.337 \mathrm{deg}$ in the $N M$ condition to $1.030 \mathrm{deg}$ in the $E H$ condition $(p=0.007)$, and to $1.035 \mathrm{deg}$ in the $F M$ condition ( $p=0.011)$. $R M S_{e}$ also significantly decreased from $1.168 \mathrm{deg}$ in $G H$ to 1.030 in $E H(p<0.001)$ and to 1.035 in $F M(p<0.001)$. No significant difference was found between the $E H$ and $F M$ conditions $(p=1.000)$. The main effect of time also introduced a significant difference in $R M S_{e}(p=0.001)$. Post-hoc analysis revealed that the $R M S_{e}$ increased significantly from $1.057 \mathrm{deg}$ in $t 1$ to $1.268 \mathrm{deg}$ in $t 4(p<0.001)$, but did not change significantly compared to $t 2(p=0.380)$ and $t 3(p=1.000)$.

Control input RMS in the roll axis is shown in Fig. 9b. No statistically significant two-way interaction was found between motion and time ( $p=0.119$ ). The main effect of motion was statistically significant. Post-hoc analysis with a Bonferroni adjustment showed that $R M S_{u}$ increased from 0.026 in $N M$ to 0.032 in $G H, E H$ and $F M(p<0.001)$. 
Table 3. Summary of main statistical test results.

\begin{tabular}{|c|c|c|c|c|c|c|c|c|c|}
\hline \multirow[t]{2}{*}{ Measure } & \multicolumn{3}{|c|}{ motion } & \multicolumn{3}{|c|}{ time } & \multicolumn{3}{|c|}{ motion $\times$ time } \\
\hline & $d f$ & $F$ & $p$ & $d f$ & $F$ & $p$ & $d f$ & $F$ & $p$ \\
\hline$M R$ & $3.0,48.0$ & 176.680 & $<0.001$ & - & - & - & - & - & - \\
\hline \multicolumn{10}{|c|}{ Roll } \\
\hline$R M S_{e}$ & $1.2,19.5^{g g}$ & 12.860 & 0.001 & $1.8,28.7^{g g}$ & 9.830 & 0.001 & $1.5,24.6^{g g}$ & 2.243 & 0.137 \\
\hline$R M S_{u}$ & $3.0,48.0$ & 20.290 & $<0.001$ & $2.1,34.5^{g g}$ & 16.026 & $<0.001$ & $3.2,51.4^{g g}$ & 2.016 & 0.119 \\
\hline$K_{p}$ & $2.0,26.3^{g g}$ & 2.839 & 0.076 & $2.0,26.0^{g g}$ & 14.259 & $<0.001$ & $4.8,62.1^{g g}$ & 1.296 & 0.278 \\
\hline$K_{v}$ & $3.0,39.0$ & 19.800 & $<0.001$ & $1.3,16.5^{g g}$ & 43.550 & $<0.001$ & $4.2,54.3^{g g}$ & 1.359 & 0.259 \\
\hline$\tau_{v}$ & $3.0,39.0$ & 16.543 & $<0.001$ & - & - & - & - & - & - \\
\hline$\omega_{n}$ & $1.7,22.5^{g g}$ & 0.658 & 0.506 & $2.0,26.5^{g g}$ & 25.879 & $<0.001$ & $2.3,30.2^{g g}$ & 2.072 & 0.137 \\
\hline$\zeta_{n}$ & $1.6,20.8^{g g}$ & 6.216 & 0.011 & $3.0,39.0$ & 2.013 & 0.128 & $9.0,117.0$ & 1.741 & 0.087 \\
\hline$\omega_{c}$ & $1.9,28.4^{g g}$ & 8.736 & 0.001 & $3.0,45.0$ & 18.182 & $<0.001$ & $9.0,135.0$ & 1.873 & 0.061 \\
\hline$\varphi_{m}$ & $1.8,27.4^{g g}$ & 7.466 & 0.003 & $1.8,27.0^{g g}$ & 5.866 & 0.009 & $1.9,29.5^{g g}$ & 2.216 & 0.128 \\
\hline \multicolumn{10}{|c|}{ Pitch } \\
\hline$R M S_{e}$ & $1.4,22.4^{g g}$ & 18.625 & $<0.001$ & $3.0,48.0$ & 11.966 & $<0.001$ & $9.0,144.0$ & 3.285 & 0.001 \\
\hline$R M S_{u}$ & $1.5,24.8^{g g}$ & 28.345 & $<0.001$ & $3.0,48.0$ & 37.400 & $<0.001$ & $4.5,72.6^{g g}$ & 7.221 & $<0.001$ \\
\hline$K_{p}$ & $3.0,21.0$ & 1.234 & 0.322 & $3.0,21.0$ & 84.871 & $<0.001$ & $3.4,23.9^{g g}$ & 2.615 & 0.068 \\
\hline$K_{v}$ & $3.0,48.0$ & 24.727 & $<0.001$ & $3.0,48.0$ & 15.849 & $<0.001$ & $9.0,144.0$ & 2.253 & 0.022 \\
\hline$\tau_{v}$ & $3.0,48.0$ & 69.213 & $<0.001$ & - & - & - & - & - & - \\
\hline$\omega_{n}$ & $1.5,23.5^{g g}$ & 8.740 & 0.003 & $1.4,22.2^{g g}$ & 27.352 & $<0.001$ & $3.9,30.2^{g g}$ & 3.389 & 0.015 \\
\hline$\zeta_{n}$ & $2.0,33.1^{g g}$ & 7.439 & 0.002 & $1.9,30.9^{g g}$ & 2.473 & 0.103 & $2.1,34.1^{g g}$ & 2.198 & 0.124 \\
\hline$\omega_{c}$ & $3.0,30.0$ & 11.389 & $<0.001$ & $3.0,30.0$ & 56.979 & $<0.001$ & $9.0,90.0$ & 2.956 & 0.004 \\
\hline$\varphi_{m}$ & $3.0,27.0$ & 4.174 & 0.015 & $3.0,27.0$ & 36.403 & $<0.001$ & $9.0,81.0$ & 3.376 & 0.001 \\
\hline
\end{tabular}

No significant changes were found between the other levels $(p=1.000)$. The main effect of time was also statistically significant for the roll control input RMS ( $p<0.001) . R M S_{u}$ increased significantly from 0.029 in $t 1$ and $t 2$ to 0.032 in $t 3$ and $t 4(p=0.003)$.

Pitch error and control input RMS are shown in Fig. 10. Fig. 10a depicts the RMS of the error in the pitch axis. A statistically significant two-way interaction was found between motion and time $(p=0.001)$. Simple main effects of motion revealed that, at time $t 1, R M S_{e}$ significantly decreased from 0.846 in $N M$ to 0.745 in $G H(p=0.001)$, to 0.723 in $E H(p<0.001)$, and to 0.738 in $F M(p=0.003)$. Also at time $t 1$, there was no significant change in $R M S_{e}$ between $G H$ and $E H(p=0.882)$ and $F M(p=1.000)$. Furthermore, no statistically significant difference was found between $E H$ and $F M(p=1.000)$. At time $t 2$, pitch error RMS significantly decreased from 1.009 in $N M$ to 0.776 in $G H$ ( $p=0.001)$, to 0.794 in $E H(p=0.017)$ and to 0.745 in $F M(p<0.001)$. There was no significant difference between $G H$ and $E H(p=1.000)$ and $F M(p=1.000)$. Also at time segment $t 2$, there was no significant difference between $E H$ and $F M(p=1.000)$. At $t 3$ no significant change was present between $N M$ and $G H(p=0.079) . R M S_{e}$ decreased significantly from 1.019 in $N M$ to 0.789 in $E H(p=0.008)$, and to 0.801 in $F M(p=0.019)$. No significant changes were present between $G H$ and $E H(p=0.978)$, and $F M(p=0.775)$. Lastly, at $t 3$, there was no significant change between $E H$ and $F M(p=1.000)$. In the last time segment $t 4$, pitch $R M S_{e}$ did not significantly change between $N M$ and $G H(p=1.000)$, but significantly decreased from 0.996 in $N M$ to 0.826 in $G H(p=0.020)$ and did not significantly change between $N M$ and $F M(p=0.560)$. Pitch error RMS significantly decreased from 0.928 in $G H$ to 0.826 in $E H(p=0.005)$ and did not significantly change between $G H$ and $F M(p=1.000)$. There was no significant change between $E H$ and $F M(p=0.081)$. Simple main effects 
of time revealed that for $N M$ there was a significant increase in pitch error RMS from 0.846 at $t 1$ to 1.009 at $t 2$ ( $p=0.043)$, to 1.019 at $t 3(p=0.014)$ and to 0.996 at $t 4(p<0.001) . R M S_{e}$ did not significantly change from $t 2$ to $t 3(p=1.000)$ and to $t 4(p=1.000)$. There was also no significant change between time segments $t 3$ and $t 4(p=1.000)$. For the $G H$ motion condition, $R M S_{e}$ did not significantly change between $t 1$ and $t 2(p=1.000)$, significantly increased from 0.745 in $t 1$ to 0.843 in $t 3(p=0.047)$, and to 0.928 in $t 4(p<0.001)$. There was no significant difference between $t 2$ and $t 3(p=0.422)$; however, pitch error RMS increased from 0.776 in $t 2$ to 0.928 in $t 4(p=0.006)$. No significant difference was found between $t 3$ and $t 4(p=0.205)$. For the $E H$ motion condition, $R M S_{e}$ significantly increased from 0.723 in $t 1$ to 0.826 in $t 4(p=0.015)$. No other significant differences were found. For the $F M$ condition, pitch error RMS did not significantly change between $t 1$ and $t 2(p=1.000)$ and $t 3(p=0.376)$, but significantly increased from 0.738 in $t 1$ to 0.916 in $t 4(p<0.001)$. Pitch error RMS also significantly increased from 0.745 in $t 2$ to 0.916 in $t 4(p=0.004)$, but there was no significant difference between $t 2$ and $t 3(p=0.358)$. There were also no significant differences between $t 3$ and $t 4(p=0.064)$.

Pitch control input RMS is depicted in Fig. 10b. There was a significant two-way interaction between motion condition and time segment $(p<0.001)$. Simple main effects of motion showed that, at $t 1, R M S_{u}$ did not significantly change between $N M$ and $G H(p=0.671)$, and $E H(p=1.000)$, but significantly increased from 0.017 in $N M$ to 0.21 in $F M(p<0.001)$. Pitch control input RMS did not significantly change at $t 1$ between $G H$ and $E H$ ( $p=0.170$ ), but significantly increased from 0.018 in $G H$ to 0.021 in $F M(p<0.001)$. There was also a significant increase from 0.017 in the $E H$ condition to 0.021 in the $F M$ condition $(p<0.001)$. At $t 2$, there were no significant differences between $N M$ and $G H(p=1.000)$ and $E H(p=1.000)$. There was a significant increase in pitch $R M S_{u}$ from 0.019 in $N M$ to 0.021 in $F M(p=0.01)$. There were no significant differences between $G H$ and $E H$ $(p=1.000)$, but there was a significant increase from 0.018 in $G H$ to 0.021 in $F M(p<0.001)$. Also at $t 2$, there was a significant increase from 0.018 in $E H$ to 0.021 in $F M(p=0.001)$. At $t 3$, there was no significant difference between $N M$ and $G H(p=0.001)$ and $E H(p=0.001)$. There was a significant increase from 0.022 in $N M$ to 0.025 in $F M(p=0.029)$. There was no significant difference in $R M S_{u}$ between $G H$ and $E H(p=1.000)$, but there was a significant increase from 0.21 in $G H$ to 0.025 in $F M(p<0.001)$. Pitch control input RMS increased significantly from 0.021 in $E H$ to 0.025 in $F M(p=0.001)$. At the last time segment, $t 4$, there was no significant difference between $N M$ and $G H(p=1.000)$, and $E H(p=0.907)$. There was a significant increase from 0.019 in $N M$ to 0.026 in $F M(p=0.001)$. Pitch control input RMS significantly increased at $t 4$ from 0.020 in $G H$ to 0.026 in $F M(p<0.001)$. No significant differences were found between $G H$ and $E H(p=1.000)$. Pitch control input RMS increased from 0.021 in $E H$ to 0.026 in $F M(p<0.001)$. Simple main effects of time showed that for the $N M$ condition, $R M S_{u}$ did not significantly change from $t 1$ to $t 2(p=0.078)$, but significantly increased from 0.017 in $t 1$ to 0.022 in $t 3(p<0.001)$ and to 0.019 in $t 4(p=0.024) . R M S_{u}$ significantly increased from 0.019 in $t 2$ to 0.022 in $t 3(p=0.001)$, but did not significantly change compared to $t 4(p=1.000)$. There was a significant decrease from 0.022 in $t 3$ to 0.019 in $t 4$ ( $p=0.007)$. In the $G H$ condition, pitch $R M S_{u}$ did not significantly change between $t 1$ and $t 2(p=1.000)$, but increased from 0.018 at $t 1$ to 0.021 at $t 3(p<0.001)$ and to 0.020 at $t 4(p=0.016)$. Pitch control input RMS increased from 0.018 in $t 2$ to 0.021 in $t 3(p<0.001)$ and to 0.020 in $t 4(p=0.037)$. There was no significant difference in the $G H$ condition between $t 3$ and $t 4(p=0.384)$. For the $E H$ condition, no significant difference was found between $t 1$ and $t 2(p=1.000)$; however, $R M S_{u}$ significantly increased from 0.017 in $t 1$ to 0.021 in $t 3(p<0.001)$ and to 0.021 in $t 4(p=0.001) . R M S_{u}$ significantly increased from 0.018 at $t 2$ to 0.021 at $t 3$ $(p<0.001)$ and to 0.021 at $t 4(p=0.029)$. There was no significant change in the $E H$ condition between $t 3$ and $t 4$ ( $p=1.000)$. Finally, in the $F M$ condition, $R M S_{u}$ did not significantly change between $t 1$ and $t 2(p=0.369)$, but significantly increased from 0.21 in $t 1$ to 0.025 in $t 3(p<0.001)$ and to 0.026 in $t 4(p<0.001)$. Pitch control input RMS significantly increased from 0.021 in $t 2$ to 0.025 in $t 3(p<0.001)$ and to 0.026 in $t 4(p<0.001)$. There was no significant difference between $t 3$ and $t 4(p=0.427)$.

\section{Pilot Model Parameters}

Pilot model parameters in the roll axis are shown in Fig. 11. Roll position gain is shown in Fig. 11a. No statistically significant two-way interaction was found between motion condition and time for the roll position gain $(p=0.278)$. The main effect of motion showed no statistically significant difference $(p=0.076)$. The main effect of time showed a statistically significant change in $K_{p}(p<0.001)$. Post-hoc analysis with a Bonferroni adjustment indicated that roll position gain did not significantly change between 0.014 in $t 1$ and 0.016 in $t 2(p=0.741)$ or $0.012 t 3(p=0.204)$, but significantly decreased to 0.011 in $t 4(p<0.001)$. Position gain also significantly decreased from 0.016 in $t 2$ to 0.012 in $t 3(p=0.009)$ and to 0.011 in $t 4(p=0.001)$. There was no statistically significant difference in roll position gain between times $t 3$ and $t 4$.

The roll velocity gain is depicted in Fig. 11b. There was no significant two-way interaction between motion 


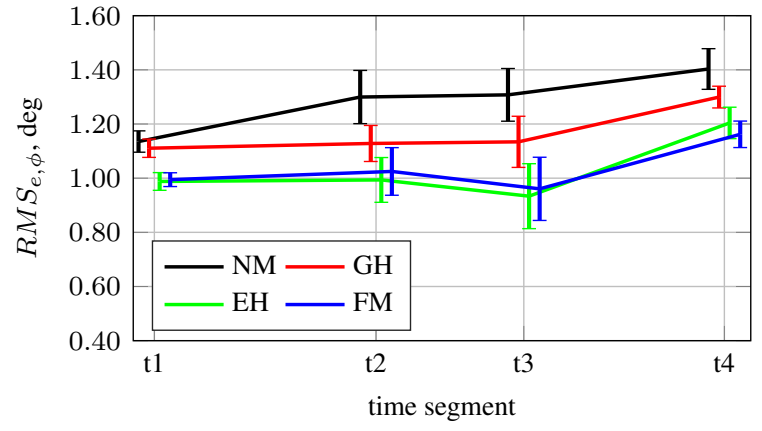

(a) Error RMS

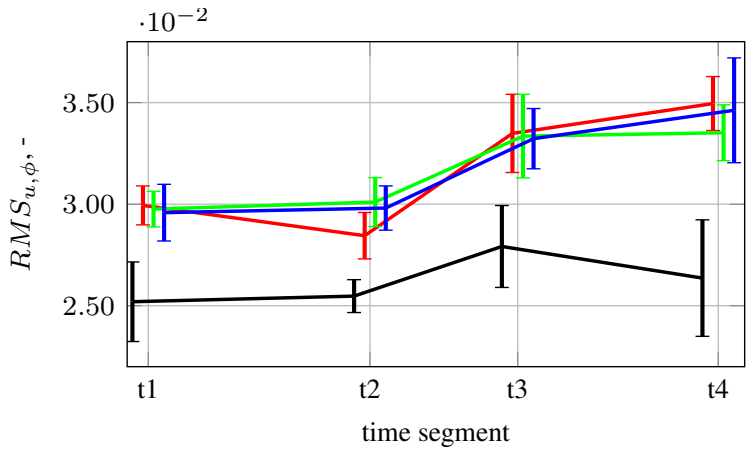

(b) Control input RMS.

Figure 9. Roll error and control input RMS.

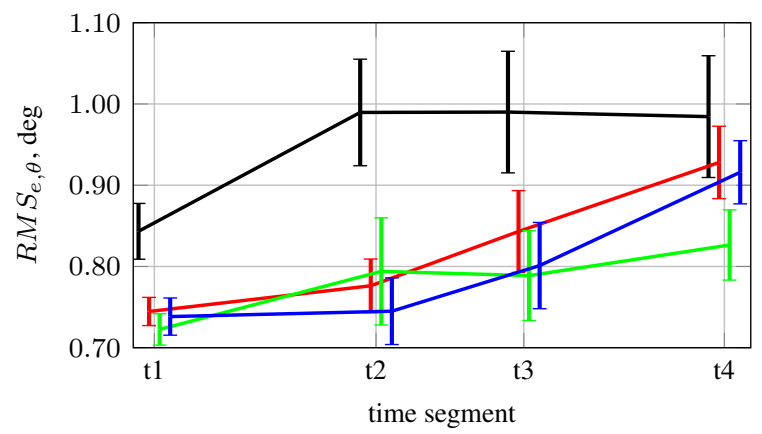

(a) Error RMS.

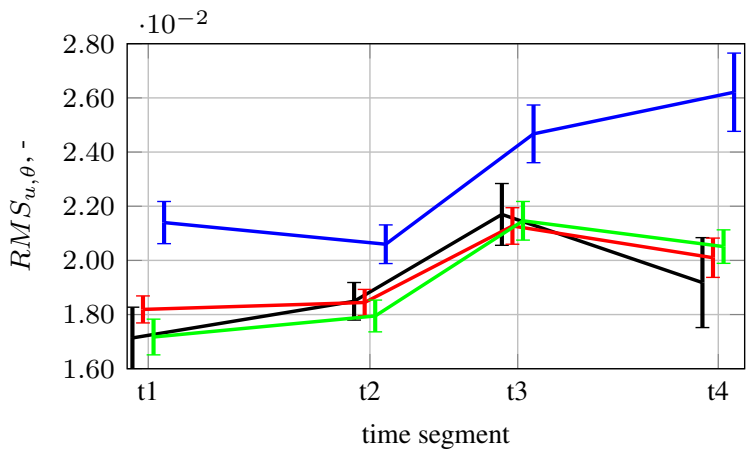

(b) Control input RMS.

Figure 10. Pitch error and control input RMS.

condition and time for the roll velocity gain. Main effect of motion showed a statistically significant difference $(p<$ 0.001). Post-hoc analysis with a Bonferroni adjustment showed that the roll velocity gain increased from 0.009 in $N M$ to 0.011 in $G H(p=0.001)$, to 0.013 in $E H(p<0.001)$, and to 0.012 in $F M(p=0.001)$. $K_{v}$ significantly increased from 0.011 in $G H$ to 0.013 in $E H$ ( $p=0.021$ ), but did not significantly change compared to $F M$. The roll velocity gain did not significantly change between $E H$ and $F M$. Main effects of time also showed a statistically significant change. Post-hoc analysis revealed that $K_{v}$ increased from 0.011 in $t 1$ to 0.015 in $t 2(p=0.002)$, decreased significantly to 0.010 in $t 3(p=0.03)$, and to 0.009 in $t 4(p<0.001)$. Roll velocity gain decreased from 0.015 in $t 2$ to 0.010 in $t 3(p<0.001)$ and to 0.009 in $t 4(p<0.001)$. It also decreased significantly from 0.010 in $t 3$ to 0.009 in $t 4(p=0.001)$.

For the neuromuscular frequency $\omega_{n}$ in Fig. 11c, no significant two-way interaction between motion and time was found $(p=0.137)$. The main effect of motion did not show a significant difference in the neuromuscular frequency $(p=0.506)$. However, the main effect of time introduced a significant difference $(p<0.001)$. Post-hoc analysis with Bonferroni adjustment revealed a significant decrease from $5.4 \mathrm{rad} / \mathrm{s}$ in $t 1$ to $4.86 \mathrm{rad} / \mathrm{s}$ in $t 2(p<0.001)$ and to 4.97 in $t 3(p=0.002)$. No significant difference was found between $t 1$ and $t 4(p=0.577)$. The neuromuscular frequency did also not change significantly between $t 2$ and $t 3(p=1.000)$. It increased significantly from $4.86 \mathrm{rad} / \mathrm{s}$ in $t 2$ to 5.57 $\mathrm{rad} / \mathrm{s}$ in $t 4(p<0.001) . \omega_{n}$ increased significantly from $4.97 \mathrm{rad} / \mathrm{s}$ in $t 3$ to $5.57 \mathrm{rad} / \mathrm{s}$ in $t 4(p<0.001)$.

The neuromuscular damping ratio is shown in Fig. 11d. No significant two-way interaction between motion condition and time existed $(p=0.087)$. The main effect of time was also not significant $(p=0.128)$. There was a significant main effect of motion $(p=0.011)$. Post-hoc analysis with Bonferroni adjustment showed that the neuromuscular damping ratio decreased significantly from 0.310 in the $N M$ condition to 0.251 in the $G H$ condition ( $p=0.03)$, but did not change significantly compared to the $E H(p=1.000)$ and $F M(p=0.860)$ conditions. Neuromuscular damping ratio increased significantly from 0.251 in $G H$ to 0.293 in $E H$ ( $p=0.001$ ), but did not change significantly compared to $F M(p=0.159)$. There was no significant difference in $\zeta_{n}$ between the $E H$ and 


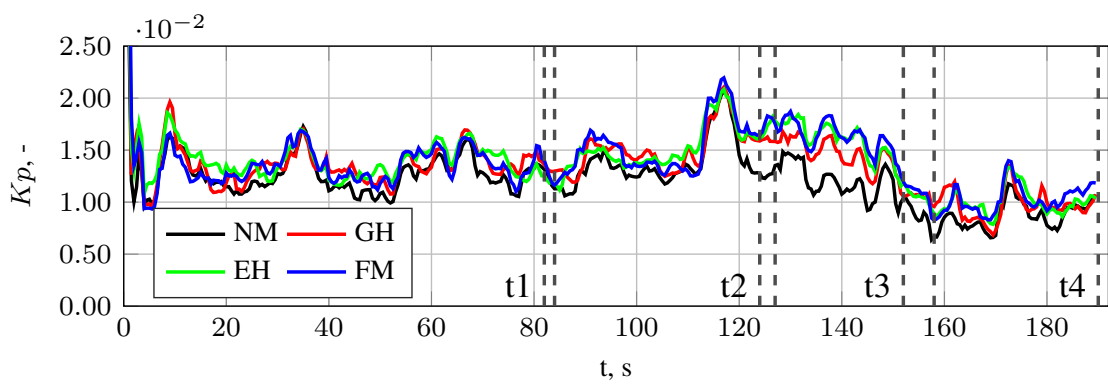

(a) Position gain.

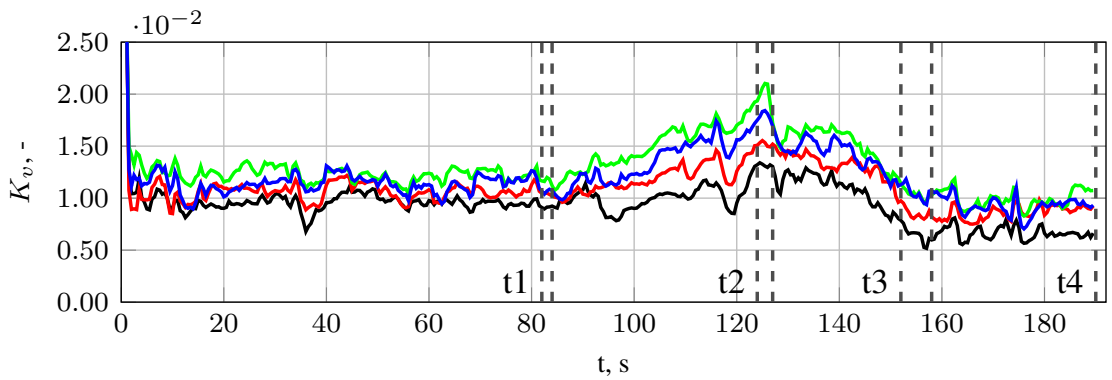

(b) Velocity gain.

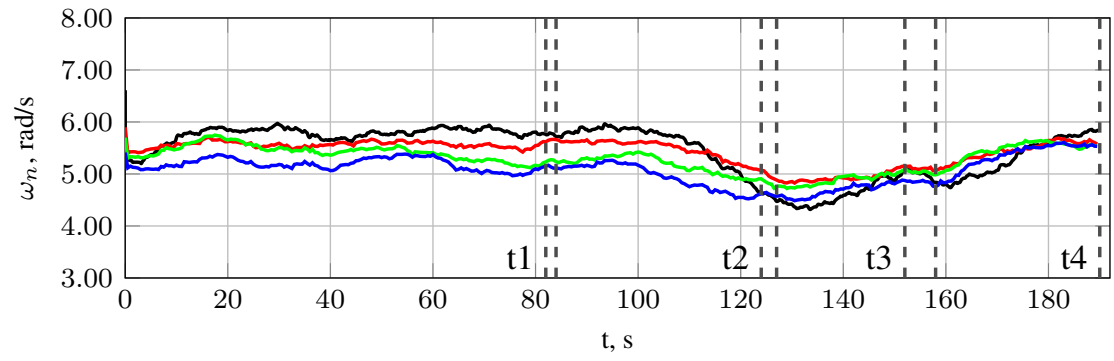

(c) Neuromuscular frequency.

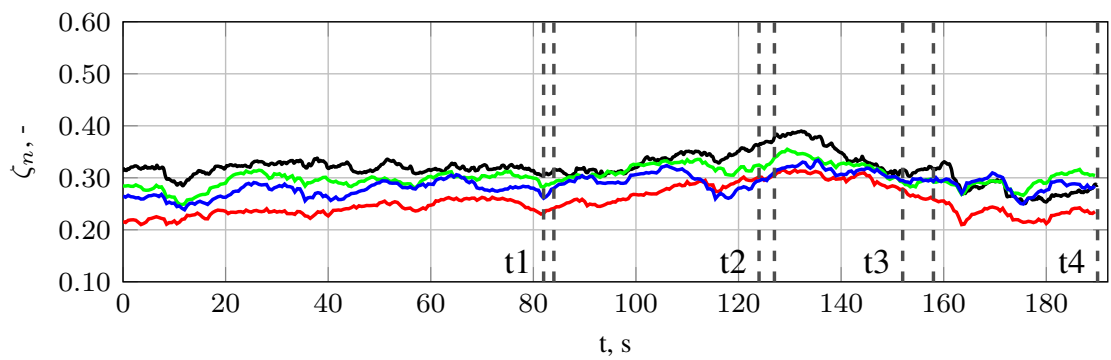

(d) Neuromuscular damping ratio.

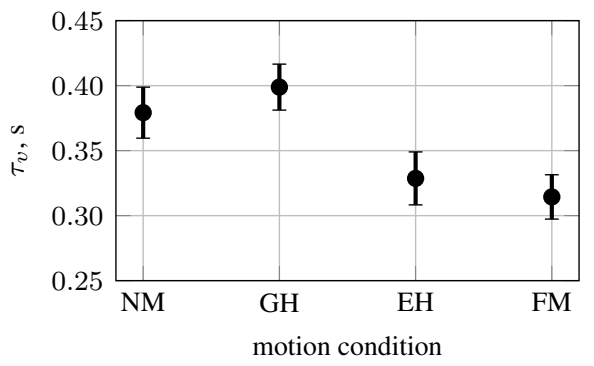

(e) Time delay.

Figure 11. Roll pilot model parameters.
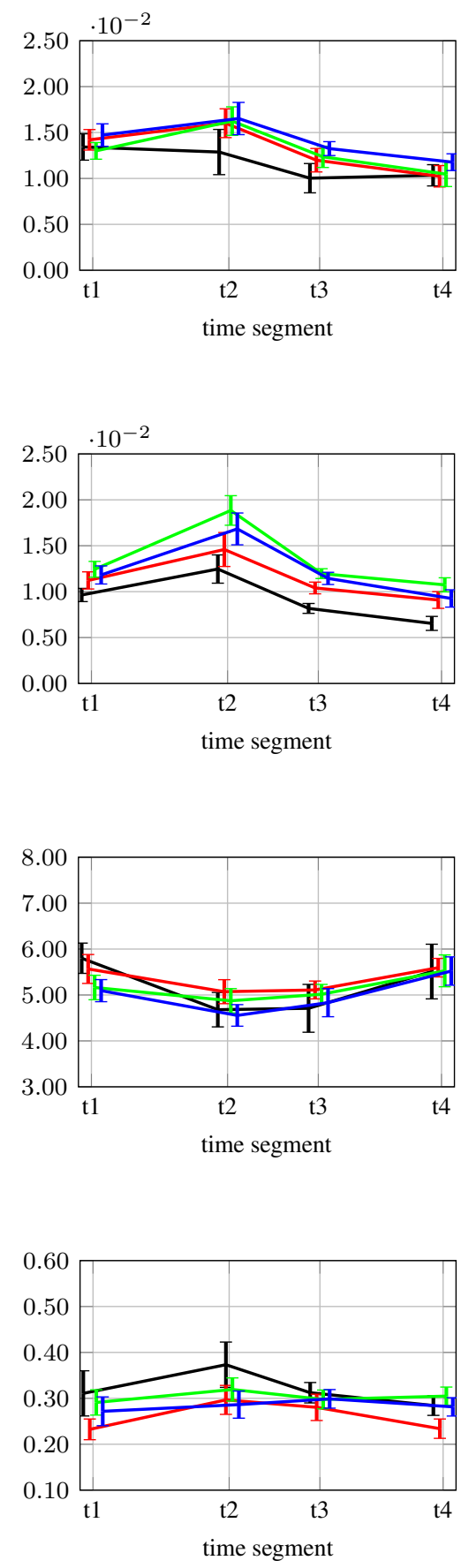
FM motion conditions.

Fig. 11e depicts the pilot roll time delay. The roll time delay significantly changed with motion, as indicated by the one-way ANOVA $(p<0.001)$. For the time delay in the roll axis, post-hoc analysis with Bonferroni adjustment revealed no significant change between the $N M$ and $G H$ conditions $(p=0.152)$. Time delay significantly decreased from $0.374 \mathrm{~s}$ in $N M$ to $0.316 \mathrm{~s}$ in $E H(p=0.034)$ and to $0.309 \mathrm{~s}$ in $F M(p=0.019)$. There was a significant decrease from $0.4 \mathrm{~s}$ in $G H$ to $0.316 \mathrm{~s}$ in $E H(p=0.001)$ and to $0.309 \mathrm{~s}$ in $F M(p<0.001)$. There was no significant change in time delay between $E H$ and $F M(p=1.000)$.

Pitch pilot model parameters are depicted in Fig. 12. There was no significant two-way interaction between motion condition and time for the pitch position gain $K_{p}(0.068)$. The main effect of motion did also not introduce any significant effects $(p=0.322)$. The main effect of time was statistically significant $(p<0.001)$. Post-hoc analysis with Bonferroni adjustment revealed that pitch position gain did not significantly change between $t 1$ and $t 2$ ( $p=0.265)$, but significantly decreased from 0.012 at $t 1$ to 0.002 at $t 3(p<0.001)$ and to 0.009 at $t 4(p=0.001)$. $K_{p}$ also significantly decreased from 0.015 at $t 2$ to 0.002 at $t 3(p<0.001)$ and to 0.006 at $t 4(p=0.007)$. Lastly, the pitch position gain significantly increased from 0.002 at $t 3$ to 0.009 at $t 4(p<0.001)$.

There was a significant two-way interaction between motion condition and time for the pitch velocity gain $(p=$ 0.022). The simple main effect of motion indicated that $K_{v}$ in pitch did not significantly change at $t 1$ between $N M$ and $G H(p=0.216)$, and $E H(p=1.000)$. It significantly increased from 0.009 in $N M$ to 0.012 in $F M(p=0.001)$. The pitch velocity gain significantly decreased from 0.11 in $G H$ to 0.009 in $E H$ for $t 1$ ( $p=0.003)$. There was a significant increase from 0.009 in $E H$ to 0.012 in $F M(p<0.001)$. For $t 2$, the pitch velocity gain did not significantly change between $N M$ and $G H(p=1.000)$ and $E H(p=1.000)$; however, it significantly increased from 0.011 in $N M$ to 0.016 in $F M(p=0.002) . K_{v}$ did not significantly change between $G H$ and $E H$ at $t 2(p=0.278)$, but it significantly increased from 0.012 in $G H$ to 0.016 in $F M(p=0.002)$. There was also a significant increase from 0.011 in $E H$ to 0.016 in $F M(p<0.001)$. At $t 3$, there was no significant change in the pitch velocity gain between $N M$ and $G H(p=0.165)$ and $E H(p=1.000)$, however it significantly increased from 0.009 in $N M$ to 0.013 in $F M$ $(p=0.001)$. There was no significant change between $G H$ and $E H(p=0.147)$ and $F M(p=0.093)$. The pitch velocity gain significantly increased from 0.009 in $E H$ to 0.013 in $F M(p<0.001)$. For the last time segment, $t 4$, $K_{v}$ did not significantly change between $N M$ and $G H(p<0.065)$ and $E H(p=1.000)$, but significantly increased from 0.009 in $N M$ to 0.012 in $F M(p<0.001) . K_{v}$ did not significantly change between $G H$ and $E H(p=0.847)$, but significantly increased from 0.010 in $G H$ to 0.012 in $F M(p=0.001)$. There was also a significant increase at $t 4$ from 0.009 in $E H$ to 0.012 in $F M(p<0.001)$. The simple main effect of time revealed that for the $N M$ condition, $K_{v}$ significantly increased from 0.009 at $t 1$ to 0.011 at $t 2$, and did not significantly change between $t 1$ and $t 3(p=1.000)$ and $t 4(p=1.000)$. The pitch velocity gain did not significantly change between $t 2$ and $t 3(p=0.103)$, but significantly decreased from 0.011 in $t 2$ to 0.009 in $t 4(p=0.046)$. Also for the $N M$ condition, there was no significant difference between $t 3$ and $t 4$. In the $G H$ condition, no significant difference in pitch velocity gain was found between $t 1$ and $t 2(p=0.696), t 3(p=1.000)$, and $t 4(p=0.569)$. There was also no significant difference between $t 2$ and $t 3(p=0.165)$, but there was a significant decrease from 0.012 in $t 2$ to 0.010 in $t 4(p=0.046)$. No significant difference was found between $t 3$ and $t 4(p=1.000)$. In the $E H$ condition, the pitch velocity gain significantly increased from 0.009 in $t 1$ to 0.011 in $t 2(p=0.006)$, but there was no significant difference between $t 1$ and $t 3(p=0.696)$ and $t 4(p=1.000)$. No other significant differences were found for the $E H$ condition between other time segments. Lastly, for the $F M$ condition, the pitch velocity gain increased from 0.012 at $t 1$ to 0.016 at $t 2(p=0.002)$, and did not significantly change between $t 1$ and $t 3(p=1.000)$ and $t 4(p=1.000)$. There was a significant decrease from 0.016 at $t 2$ to 0.013 at $t 3(p=0.001)$ and to 0.012 at $t 4(p=0.002)$. No significant difference was found between $t 3$ and $t 4$ for the $F M$ condition $(p=1.000)$.

A significant two-way interaction was found between motion condition and time for the pitch neuromuscular frequency $(p=0.015)$. The simple main effect of motion showed that at time $t 1, \omega_{n}$ did not significantly change from the $N M$ to $G H$ condition ( $p=0.873$ ), but significantly decreased from $8.544 \mathrm{rad} / \mathrm{s}$ in $N M$ to $6.549 \mathrm{rad} / \mathrm{s}$ in $E H(p<0.001)$. Also at $t 1$, there was no significant difference between $N M$ and $F M(p=0.073)$. There were no significant differences between $G H$ and $E H(p=0.106)$ and $F M(p=0.342)$. There was a significant increase in neuromuscular frequency from $6.549 \mathrm{rad} / \mathrm{s}$ in $E H$ to $12.701 \mathrm{rad} / \mathrm{s}$ in $F M(0.009)$. At time $t 2$, there was no significant difference between $N M$ and $G H(p=0.650)$, but $\omega_{n}$ decreased significantly from $7.713 \mathrm{rad} / \mathrm{s}$ in $N M$ to $6.375 \mathrm{rad} / \mathrm{s}$ in $E H(p=0.004)$. There was also a significant increase in neuromuscular frequency from $7.713 \mathrm{rad} / \mathrm{s}$ in $N M$ to $12.362 \mathrm{rad} / \mathrm{s}$ in $F M(p=0.037)$. There was no significant difference between the $G H$ and $E H$ conditions $(p=0.185)$ and the $F M$ condition $(p=0.165) . \omega_{n}$ significantly increased from $6.374 \mathrm{rad} / \mathrm{s}$ in $E H$ to $12.362 \mathrm{rad} / \mathrm{s}$ in $F M(p=0.013)$. At time $t 3$, the only significant difference found was an increase from $6.312 \mathrm{rad} / \mathrm{s}$ in $E H$ to $11.311 \mathrm{rad} / \mathrm{s}$ in $F M(p=0.023)$. In the last time segment, $t 4$, there was no significant difference between $N M$ and 


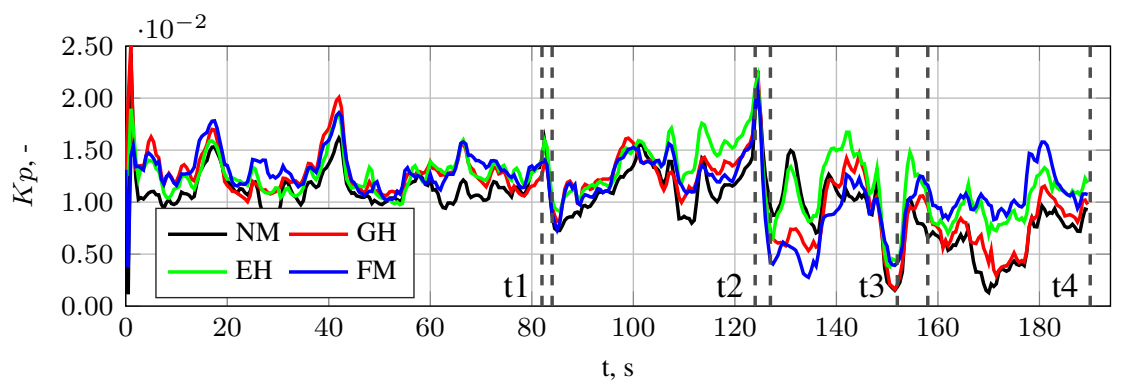

(a) Position gain.

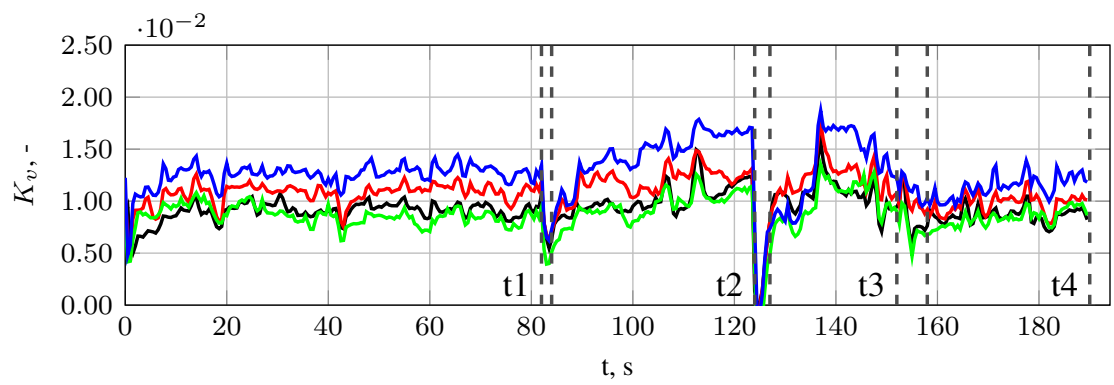

(b) Velocity gain.

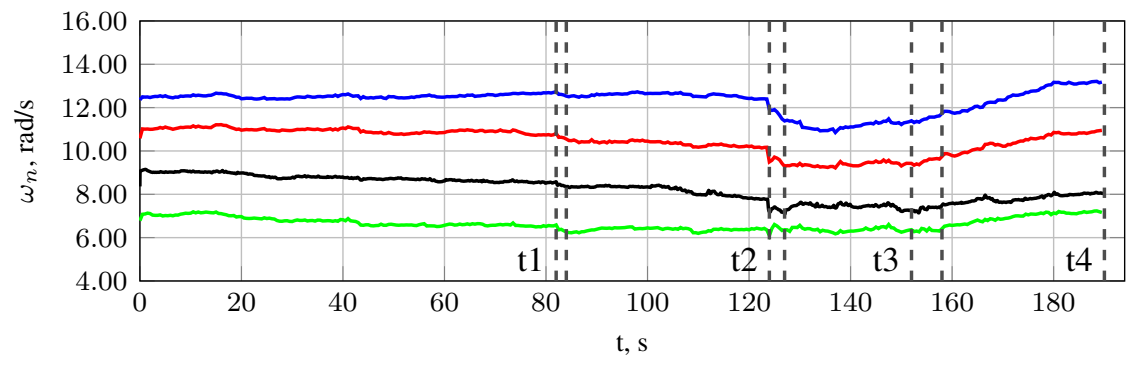

(c) Neuromuscular frequency.

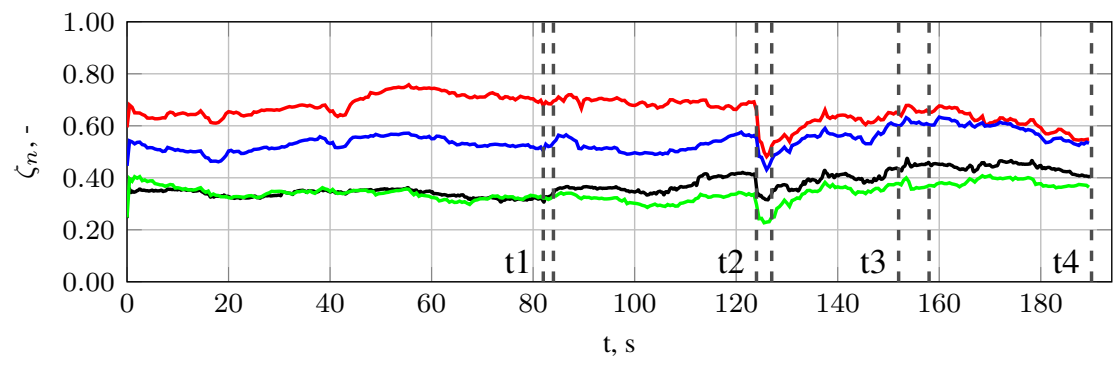

(d) Neuromuscular damping ratio.

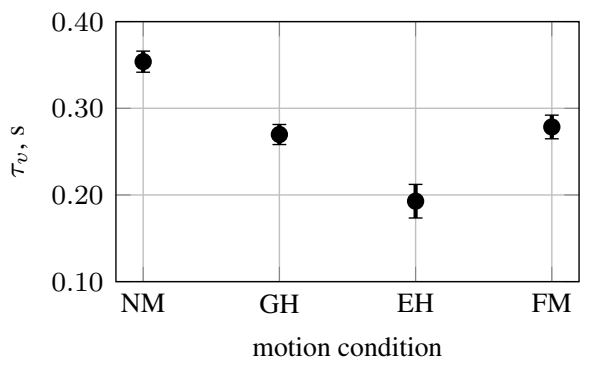

(e) Time delay.

Figure 12. Pitch pilot model parameters.
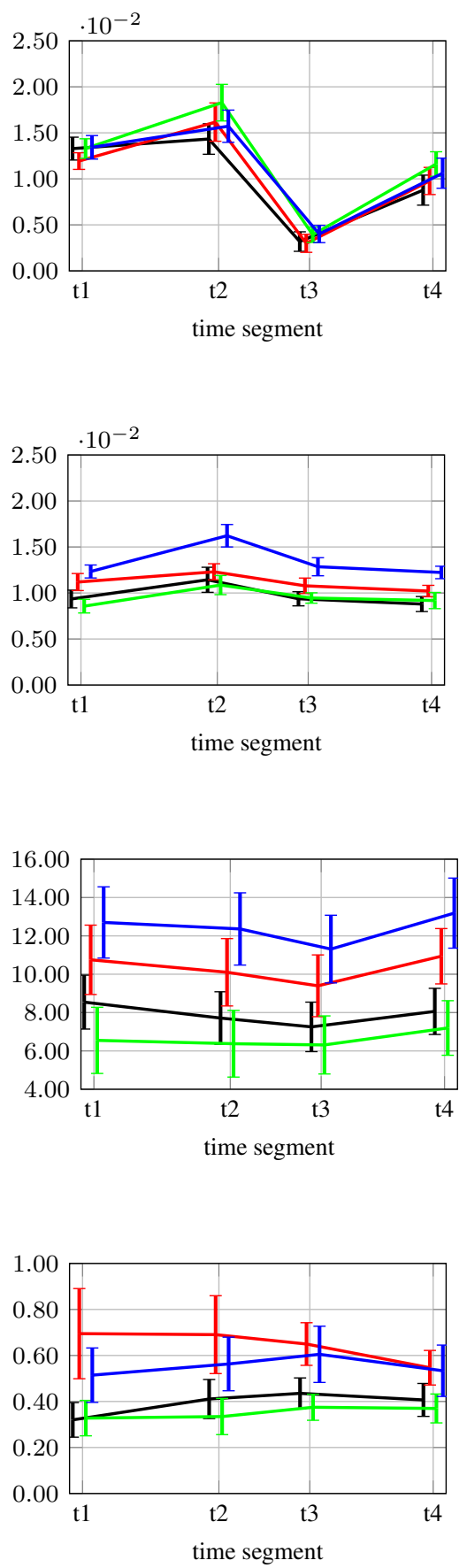
$G H(p=0.154)$. Pitch neuromuscular frequency significantly decreased from $8.061 \mathrm{rad} / \mathrm{s}$ in $N M$ to $7.196 \mathrm{rad} / \mathrm{s}$ in $E H(p=0.035)$, and significantly increased to $13.187 \mathrm{rad} / \mathrm{s}$ in $F M(p=0.010)$. $\omega_{n}$ significantly decreased from $10.937 \mathrm{rad} / \mathrm{s}$ in $G H$ to $7.196 \mathrm{rad} / \mathrm{s}$ in $E H(p=0.048)$, but did not significantly change compared to $F M(p=0.204)$. Finally, the pitch neuromuscular frequency significantly increased from $7.196 \mathrm{rad} / \mathrm{s}$ in $E H$ to $13.187 \mathrm{rad} / \mathrm{s}$ in $F M$ $(p=0.006)$. The simple main effect of time showed that in the $N M$ condition, the pitch neuromuscular frequency significantly decreased from $8.544 \mathrm{rad} / \mathrm{s}$ in $t 1$ to $7.713 \mathrm{rad} / \mathrm{s}$ in $t 2(p<0.001)$ and to $7.254 \mathrm{rad} / \mathrm{s}$ at $t 3(p<0.001)$. There was no significant difference for the $N M$ condition from time $t 1$ to time $t 4(p=0.191)$. Pitch neuromuscular frequency significantly decreased from $7.713 \mathrm{rad} / \mathrm{s}$ in $G H$ to $7.254 \mathrm{rad} / \mathrm{s}$ in $E H$ ( $p=0.015$ ), but there was no significant difference between $t 2$ and $t 4(p=0.328)$. Also for the $N M$ condition, there was a significant increase from $7.254 \mathrm{rad} / \mathrm{s}$ in $E H$ to $8.061 \mathrm{rad} / \mathrm{s}$ in $F M(p<0.001)$. For the $G H$ condition, there was a significant decrease from $10.752 \mathrm{rad} / \mathrm{s}$ at $t 1$ to $10.102 \mathrm{rad} / \mathrm{s}$ at $t 2$, but there were no significant differences between $t 1$ and $t 3(p=0.067)$ and $t 4(p=1.000)$. The pitch neuromuscular frequency was not significantly different between $t 2$ and $t 3(p=0.394)$ and $t 4(p=0.307)$. There was a significant increase from $9.396 \mathrm{rad} / \mathrm{s}$ in $t 3$ to $10.937 \mathrm{rad} / \mathrm{s} \mathrm{in} t 4(p<0.001)$. In the $E H$ condition, $\omega_{n}$ did not significantly change between $t 1$ and $t 2(p=1.000)$ and $t 3(p=1.000)$. There was a significant increase in pitch neuromuscular frequency from $6.549 \mathrm{rad} / \mathrm{s}$ in $t 1$ to $7.196 \mathrm{rad} / \mathrm{s}$ in $t 4(p=0.008)$. There was no significant difference from $t 2$ to $t 3(p=1.000)$; however, $\omega_{n}$ significantly increased from $6.375 \mathrm{rad} / \mathrm{s}$ in $t 2$ to $7.196 \mathrm{rad} / \mathrm{s}$ in $t 4(p=0.003)$. There was also a significant increase in the $E H$ condition from $6.312 \mathrm{rad} / \mathrm{s}$ in $t 3$ to $7.196 \mathrm{rad} / \mathrm{s}$ in $t 4(p<0.001)$. Finally, for the $F M$ motion condition, the pitch neuromuscular frequency did not significantly change from $t 1$ to $t 2(p=0.534)$, but significantly decreased from $12.701 \mathrm{rad} / \mathrm{s}$ in $t 1$ to $11.311 \mathrm{rad} / \mathrm{s}$ in $t 3(p=0.005)$. There was no significant difference between $t 1$ and $t 4(p=0.753)$. The pitch neuromuscular frequency significantly decreased from $12.362 \mathrm{rad} / \mathrm{s}$ at $t 2$ to $11.311 \mathrm{rad} / \mathrm{s}$ in $t 3(p=0.004)$, but did not significantly change compared to $t 4(p=0.063)$. Lastly, $\omega_{n}$ significantly increased from $11.311 \mathrm{rad} / \mathrm{s}$ in $t 3$ to $13.187 \mathrm{rad} / \mathrm{s}$ in $t 4$ for motion condition $F M(p<0.001)$.

There was no significant two-way interaction between motion condition and time for the neuromuscular damping ratio in the pitch axis $(p=0.124)$. The main effect of motion was statistically significant $(p=0.002)$. Post-hoc analysis with a Bonferroni adjustment revealed that $\zeta_{n}$ significantly increased from 0.393 in $N M$ to 0.645 in $G H$ $(p=0.029)$, but was not significantly different between $N M$ and $E H(p=0.858)$ and $F M(p=0.213)$. There was also a significant decrease from 0.645 in the $G H$ motion condition to 0.352 in the $E H$ motion condition $(p=0.006)$, but no difference between the $G H$ and $F M$ conditions $(p=1.000)$. Finally, there was no significant difference in pitch neuromuscular damping ratio between the $E H$ and $F M$ conditions ( $p=0.053)$.

There was a significant difference introduced by motion for the pilot pitch time delay. Post-hoc analysis with Bonferroni adjustment revealed a decrease from $0.354 \mathrm{~s}$ in the $N M$ condition to $0.270 \mathrm{~s}$ in $G H(p<0.001)$, to 0.193 $\mathrm{s}$ in $E H(p<0.001)$ and to $0.278 \mathrm{~s}$ in $F M(p<0.001)$. The time delay also significantly decreased from $0.270 \mathrm{~s}$ in $G H$ to $0.193 \mathrm{~s}$ in $E H(p<0.001)$, but did not significantly change compared to the $F M$ condition $(p=1.000)$. The pitch time delay significantly increased from $0.193 \mathrm{~s}$ in the $E H$ condition to $0.278 \mathrm{~s}$ in the $F M$ condition $(p<0.001)$.

\section{Aircraft Dynamics}

Identified roll and pitch aircraft dynamics parameters are shown in Fig. 13 and Fig. 14, respectively. As these aircraft dynamics parameters were estimated with the end goal of determining the time-varying open-loop parameters presented in Section E, no ANOVA was performed. From Figures 13a and 14a, it can be seen that the dynamics gain in both roll and pitch was lower in $t 2$ and $t 3$; that is, close to the stall point and at the end of the dive. This is in line with the observed reduced control authority around these time segments. The roll dynamics break-frequency first increased from $t 1$ to $t 2$ after which it decreased in $t 3$ and increased again in $t 4$ (Fig. 13b). The pitch dynamics break-frequency decreased from $t 1$ to $t 2$ and then increased slightly in $t 3$ and increased further in $t 4$ (Fig. 14b). These observation indicate a slight reduction in aircraft roll stability in $t 3$, and a reduction in pitch stability mainly in $t 2$.

To verify the accuracy of the highly simplified aircraft dynamics representation in Eq. (3), the variance accounted for (VAF) of the modeled aircraft dynamics output for the first 82 seconds of flight in $S 1$ using the MLE and EKF methods is depicted in Fig. 15. The VAF is relatively high for both parameter estimation methods, indicating that the simplified dynamics can describe the non-linear aircraft model reasonably well. It can be seen that the VAF is higher using the EKF for both the roll and pitch axes. This is to be expected, since the MLE method assumes constant dynamics, whereas the EKF is able to identify time-varying parameters. 


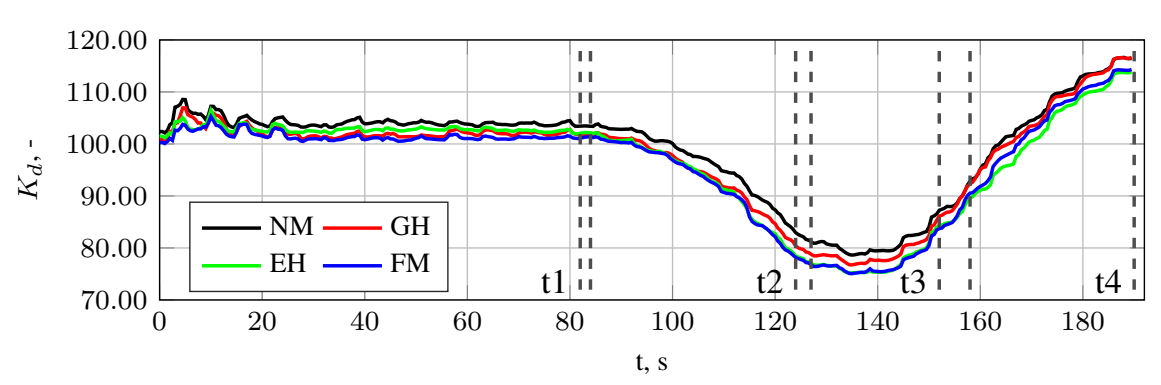

(a) Dynamics gain.

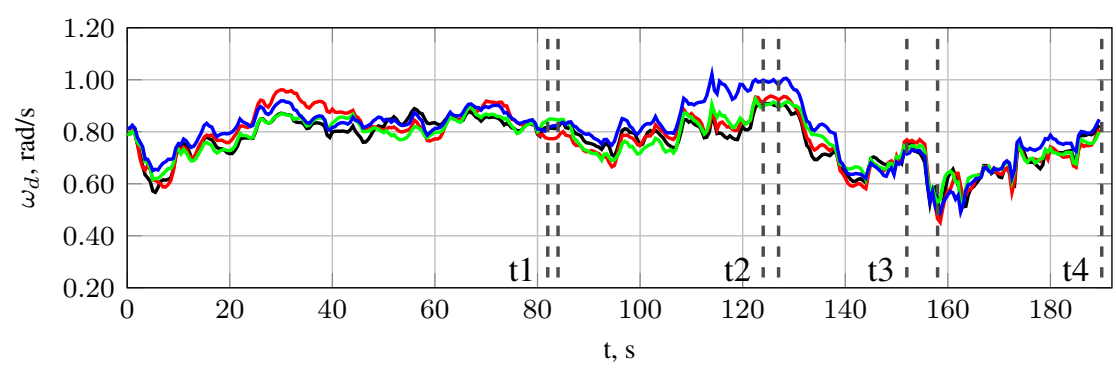

(b) Dynamics break frequency.

Figure 13. Roll dynamics characteristics.

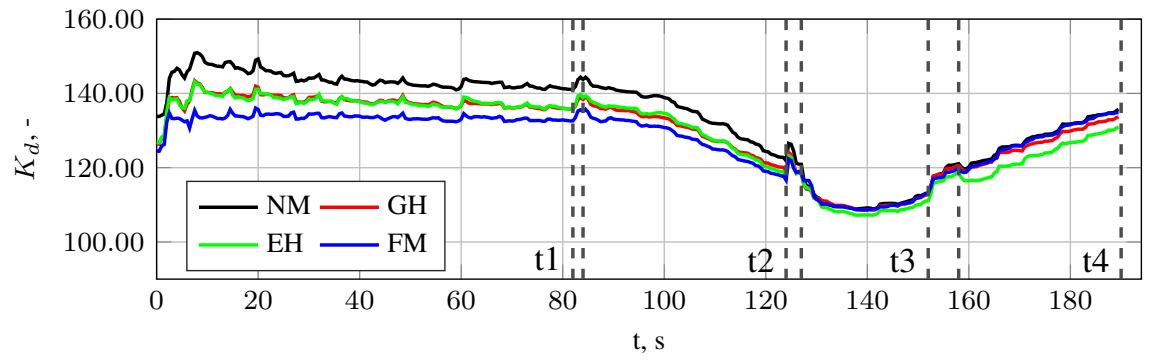

(a) Dynamics gain.

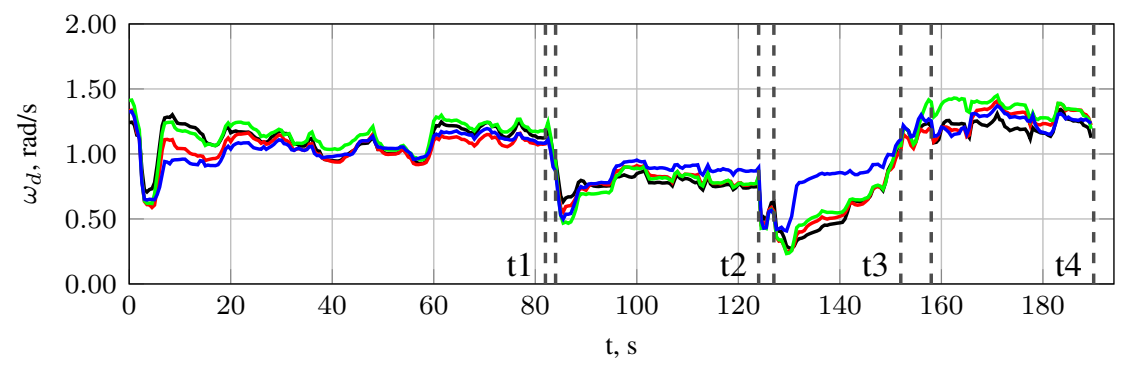

(b) Dynamics break frequency.
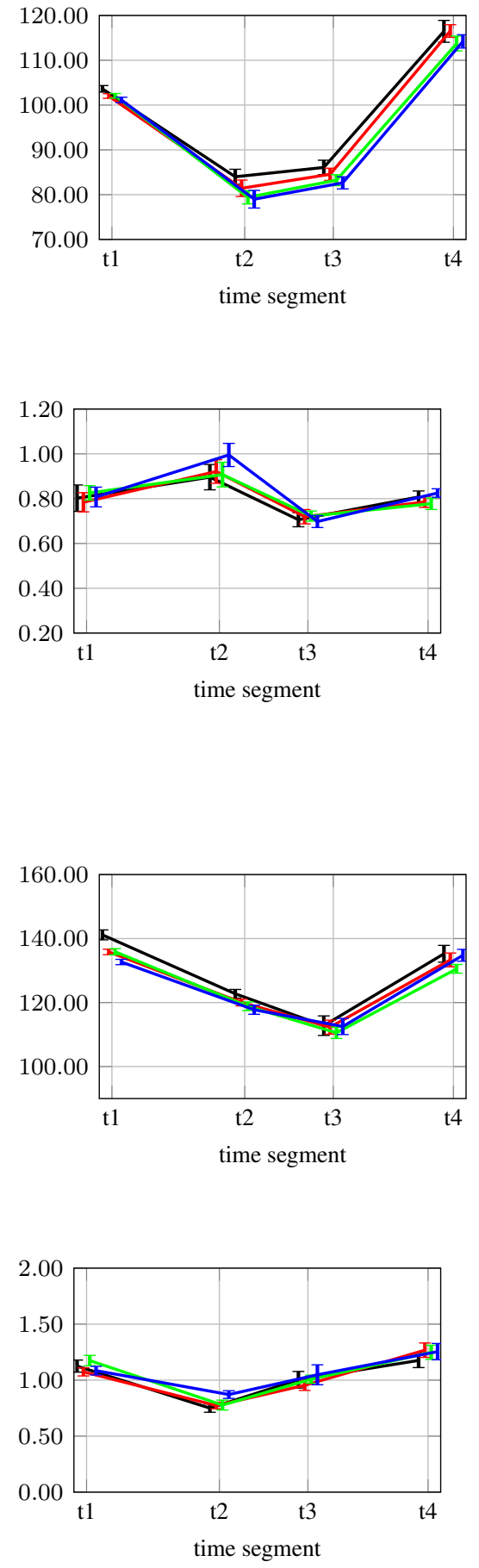

Figure 14. Pitch dynamics characteristics.

\section{E. Performance and Stability}

Performance and stability in the frequency domain are characterized by the pilot-vehicle transfer function crossover frequency and phase margin in Figures 16 and 17 for roll and pitch, respectively.

There was no significant two-way interaction between motion and time for the roll crossover frequency $(p=$ 0.061), see Table 3. The main effect of motion showed a significant result $(p=0.001)$. Post-hoc analysis with a Bonferroni adjustment revealed that $\omega_{c}$ in roll significantly increased from $1.152 \mathrm{rad} / \mathrm{s}$ in $N M$ to $1.273 \mathrm{rad} / \mathrm{s}$ in $G H$ 


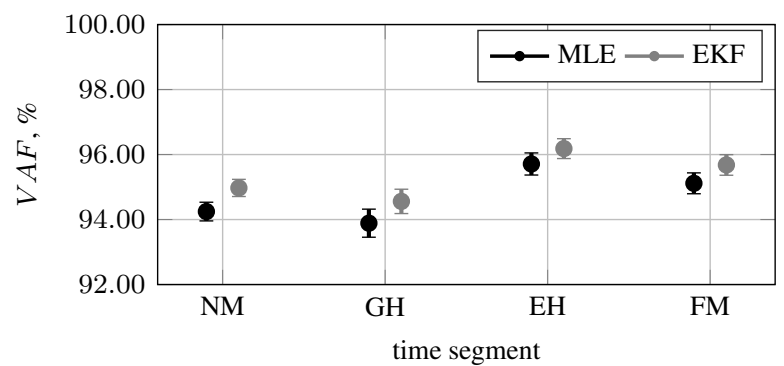

(a) Roll dynamics VAF.

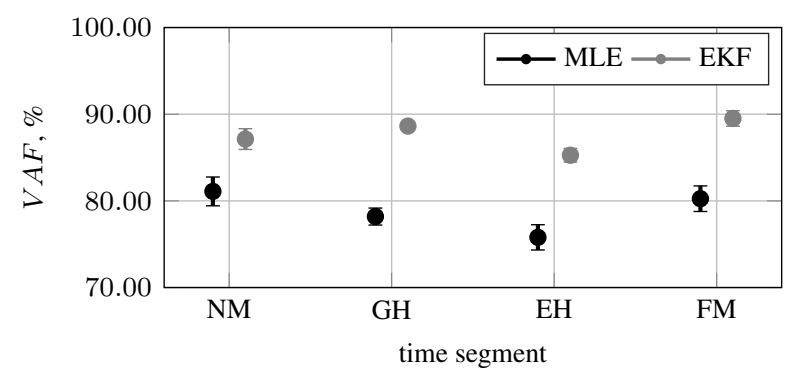

(b) Pitch dynamics VAF.

Figure 15. Variance Accounted For for aircraft identification.

( $p=0.028)$ and to $1.368 \mathrm{rad} / \mathrm{s}$ in $E H(p=0.004)$, but did not significantly change compared to $F M(p=0.082)$. There was no significant difference in roll crossover frequency between $G H$ and $E H(p=0.165)$ and $F M(p=$ 1.000), or between $E H$ and $F M(p=0.505)$. The main effect of time also showed a significant effect $(p<0.001)$. Roll crossover frequency did not significantly change between $1.381 \mathrm{rad} / \mathrm{s}$ at $t 1$ and $1.359 \mathrm{rad} / \mathrm{s}$ at $t 2(p=1.000)$, but significantly decreased from $1.381 \mathrm{rad} / \mathrm{s}$ at $t 1$ to $1.111 \mathrm{rad} / \mathrm{s}$ at $t 3(p<0.001)$ and to $1.242 \mathrm{rad} / \mathrm{s}$ at $t 4(p=0.001)$. There was also a significant decrease from $1.359 \mathrm{rad} / \mathrm{s}$ at $t 2$ to $1.111 \mathrm{rad} / \mathrm{s}$ at $t 3(p=0.002)$, but no significant difference compared to the roll crossover frequency at $t 4(p=0.153)$. Lastly, roll $\omega_{c}$ significantly increased from 1.111 at $t 3$ to $1.242 \mathrm{rad} / \mathrm{s}$ at $t 4(p=0.005)$.

In Fig. 16b, the roll phase margins are shown. There was no significant two-way interaction between motion and time $(p=0.128)$. The main effect of motion showed that there was a significant difference in roll phase margin $(p<0.003)$. Post-hoc analysis with Bonferroni adjustment revealed that roll phase margin significantly increased from $74.720 \mathrm{deg}$ in $N M$ to $79.975 \mathrm{deg}$ in $G H(p=0.029)$, and to $85.481 \mathrm{deg}$ in $E H(p<0.001)$. There was no significant difference between $N M$ and $F M(p=0.297)$. There was also a significant increase from $79.975 \mathrm{deg}$ in $G H$ to $85.481 \mathrm{deg}$ in $E H(p=0.001)$, but no significant difference between $G H$ and $F M(p=1.000)$. Roll phase margin significantly decreased from $85.481 \mathrm{deg}$ in $E H$ to $80.751 \mathrm{deg}$ in $F M(p=0.028)$. The main effect of time also showed a significant difference $(p=0.009)$. Post-hoc analysis with a Bonferroni adjustment showed that roll phase margin significantly increased from $78.821 \mathrm{deg}$ at $t 1$ to $87.578 \mathrm{deg}$ at $t 2(p=0.002)$, but did not significantly change compared to $t 3(p=1.000)$ and $t 4(p=1.000)$. The roll phase margin significantly decreased from 87.578 deg in $t 2$ to $77.871 \mathrm{deg}$ in $t 3(p=0.011)$ and to $76.657 \mathrm{deg}$ in $t 4(p=0.025)$. Finally, $\varphi_{m}$ in roll did not significantly change between $t 3$ and $t 4(p=1.000)$.

Fig. 17a shows the crossover frequency for the pitch axis. A significant two-way interaction was found between motion condition and time $(p=0.004)$. The simple main effect of motion revealed that at $t 1$, pitch crossover frequency did not significantly change from $1.465 \mathrm{rad} / \mathrm{s}$ in $N M$ to $1.519 \mathrm{rad} / \mathrm{s}$ in $G H(p=1.000)$, to $1.337 \mathrm{rad} / \mathrm{s}$ at $E H$ ( $p=0.845)$ and to $1.644 \mathrm{rad} / \mathrm{s}$ at $F M(p=0.087)$. The pitch crossover frequency significantly decreased from 1.519 $\mathrm{rad} / \mathrm{s}$ in $G H$ to $1.337 \mathrm{rad} / \mathrm{s}$ in $E H(p=0.034)$ and significantly increased to $1.644 \mathrm{rad} / \mathrm{s}$ in $F M(p=0.046)$. There was a significant increase from $1.337 \mathrm{rad} / \mathrm{s}$ in $E H$ to $1.644 \mathrm{rad} / \mathrm{s}$ in $F M$. At time $t 2$, pitch crossover frequency did not significantly change from $1.616 \mathrm{rad} / \mathrm{s}$ in $N M$ to $1.695 \mathrm{rad} / \mathrm{s}$ in $G H(p=1.000)$ or to $1.664 \mathrm{rad} / \mathrm{s}$ in $E H(p=1.000)$,

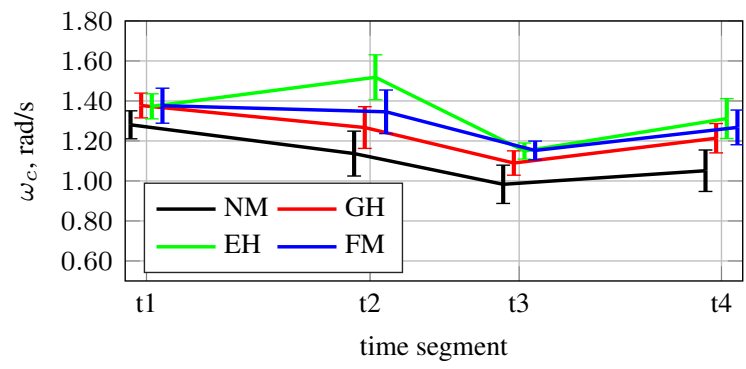

(a) Crossover frequency.

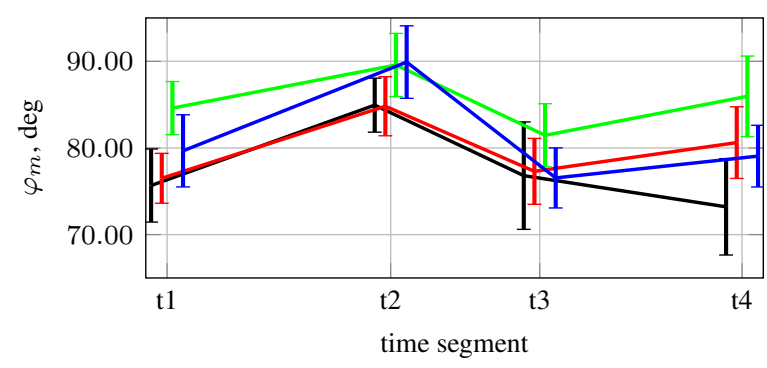

(b) Phase margin.

Figure 16. Roll performance and stability. 


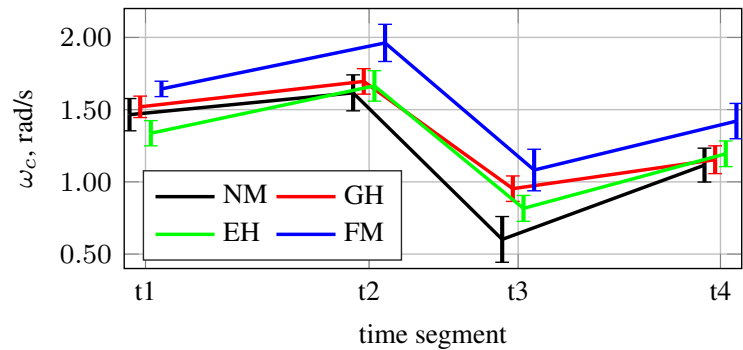

(a) Crossover frequency.

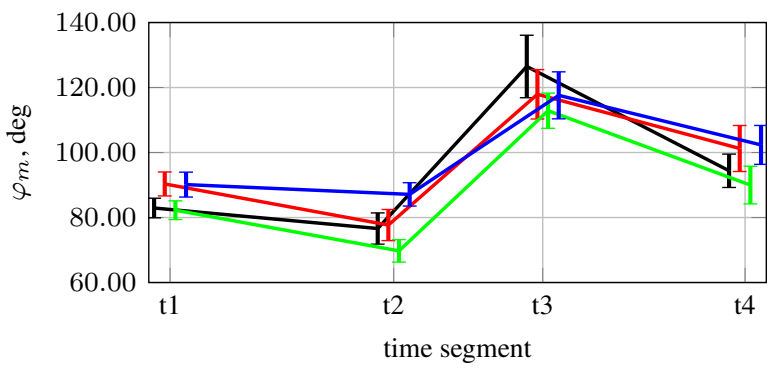

(b) Phase margin.

Figure 17. Pitch performance and stability.

but significantly increased to $1.962 \mathrm{rad} / \mathrm{s}$ in $F M(p=0.014)$. There was no significant difference between $G H$ and $E H(p=1.000)$ and $F M(p=0.063)$. There was a significant increase from $1.664 \mathrm{rad} / \mathrm{s}$ in $E H$ to $1.962 \mathrm{rad} / \mathrm{s}$ in $F M(p=0.031)$. At time segment $t 3, \omega_{c}$ in pitch significantly increased from $0.691 \mathrm{rad} / \mathrm{s}$ for $N M$ to $1.108 \mathrm{rad} / \mathrm{s}$ for $G H(p=0.021)$, but did not significantly change compared to $E H(p=0.206)$ and $F M(p=0.064)$. There was no significant difference between $G H$ and $E H(p=0.269)$ and $F M(p=1.000)$. Lastly, no significant change was found between $E H$ and $F M(p=0.175)$. At $t 4$, the pitch crossover frequency did not significantly change from $1.116 \mathrm{rad} / \mathrm{s}$ in $N M$ to $1.153 \mathrm{rad} / \mathrm{s}$ in $G H(p=1.000)$ or to $1.153 \mathrm{rad} / \mathrm{s}$ in $E H(p=1.000)$, but significantly increased to $1.421 \mathrm{rads} / \mathrm{s}$ for $F M(p=0.036)$. There was no significant difference between $G H$ and $E H(p=1.000)$. A significant increase was found between $1.153 \mathrm{rad} / \mathrm{s}$ in $G H$ and $1.421 \mathrm{rad} / \mathrm{s}$ in $F M(p=0.037)$. Pitch $\omega_{c}$ was not significantly different between $E H$ and $F M(p=0.096)$. The simple main effect of time revealed that in the $N M$ condition, the pitch crossover frequency was not significantly different between $1.499 \mathrm{rad} / \mathrm{s}$ at $t 1$ and $1.655 \mathrm{rad} / \mathrm{s}$ at $t 2$ ( $p=0.297)$, but significantly decreased to $0.596 \mathrm{rad} / \mathrm{s}$ at $t 3(p<0.001)$ and to $1.125 \mathrm{rad} / \mathrm{s}$ at $t 4(p<0.001)$. There was a significant decrease from $1.655 \mathrm{rad} / \mathrm{s}$ at $t 2$ to $0.596 \mathrm{rad} / \mathrm{s}$ at $t 3(p<0.001)$ and to $1.125 \mathrm{rad} / \mathrm{s}$ at $t 4(p=0.001)$. A significant increase was found from $0.596 \mathrm{rad} / \mathrm{s}$ at $t 3$ to $1.125 \mathrm{rad} / \mathrm{s}$ at $t 4(p=0.001)$. In the $G H$ condition, pitch $\omega_{c}$ did not significantly change from $1.530 \mathrm{rad} / \mathrm{s}$ at $t 1$ to $1.731 \mathrm{rad} / \mathrm{s}$ at $t 2(p=0.066)$, but significantly decreased to $0.942 \mathrm{rad} / \mathrm{s}$ at $t 3(p<0.001)$ and to $1.156 \mathrm{rad} / \mathrm{s}$ at $t 4(p<0.001)$. There was also a significant decrease from $1.731 \mathrm{rad} / \mathrm{s}$ at $t 2$ to $0.942 \mathrm{rad} / \mathrm{s}$ at $t 3(p<0.001)$ and to $1.156 \mathrm{rad} / \mathrm{s}$ at $t 4(p<0.001)$. No significant change was found between $t 3$ and $t 4(p=0.334)$. In the $E H$ condition, pitch crossover frequency significantly increased from $1.462 \mathrm{rad} / \mathrm{s}$ at $t 1$ to $1.718 \mathrm{rad} / \mathrm{s}$ at $t 2(p=0.023)$, significantly decreased to $0.872 \mathrm{rad} / \mathrm{s}$ at $t 3(p<0.001)$, and did not significantly change compared in $t 4(p=0.244)$. There was also a significant decrease from $1.718 \mathrm{rad} / \mathrm{s}$ at $t 2$ to $0.872 \mathrm{rad} / \mathrm{s}$ at $t 3(p<0.001)$ and to $1.310 \mathrm{rad} / \mathrm{s}$ at $t 4(p=0.001)$. Also in the $E H$ condition, pitch $\omega_{c}$ significantly increased from $0.872 \mathrm{rad} / \mathrm{s}$ at $t 3$ to $1.310 \mathrm{rad} / \mathrm{s}$ at $t 4(p=0.002)$. Lastly, in the $F M$ condition, the pitch crossover frequency significantly increased from $1.644 \mathrm{rad} / \mathrm{s}$ at $t 1$ to $1.962 \mathrm{rad} / \mathrm{s}$ at $t 2(p=0.003)$, significantly decreased to $1.055 \mathrm{rad} / \mathrm{s}$ at $t 3(p<0.001)$, and significantly decreased to $1.421 \mathrm{rad} / \mathrm{s}$ at $t 4(p=0.049)$. There was a significant decrease from $1.962 \mathrm{rad} / \mathrm{s}$ at $t 2$ to $1.055 \mathrm{rad} / \mathrm{s}$ at $t 3(p<0.001)$ and to $1.421 \mathrm{rad} / \mathrm{s}$ at $t 4(p<0.001)$. Pitch crossover frequency significantly increased from $1.055 \mathrm{rad} / \mathrm{s}$ at $t 3$ to $1.421 \mathrm{rad} / \mathrm{s}$ at $t 4(p=0.012)$.

For the pitch phase margin in Fig. 17b, there was a significant two-way interaction between motion condition and time $(p=0.001)$. The simple main effect of motion showed that at $t 1$, there was a significant difference between $82.927 \mathrm{deg}$ in $N M$ and $90.333 \mathrm{deg}$ in $G H(p=0.039)$, but no significant difference between $N M$ and $E H(p=$ $1.000)$ or $F M(p=0.067)$. There was a significant decrease from $90.333 \mathrm{deg}$ in $G H$ to 82.259 deg in $E H$ ( $p=$ $0.020)$, but no difference between $G H$ and $F M(p=1.000)$. Also at $t 1$, there was a significant increase from 82.259 deg in $E H$ to $90.131 \mathrm{deg}$ in $F M(p=0.028)$. At time $t 2$, no significant difference was found for the pitch phase margin between $76.618 \mathrm{deg}$ in $N M$ and $77.688 \mathrm{deg}$ in $G H(p=1.000)$ and $69.745 \mathrm{deg}$ in $E H(p=0.185)$. There was a significant increase from $76.618 \mathrm{deg}$ in $N M$ to $87.124 \mathrm{deg}$ in $F M(p=0.046)$. No significant difference existed between $G H$ and $E H$ ( $p=0.190$ ); however, the pitch phase margin increased from $77.688 \mathrm{deg}$ in $G H$ to $87.124 \mathrm{deg}$ in $F M(p=0.034)$. Lastly at $t 2$, there was a significant increase from $69.745 \mathrm{deg}$ in $E H$ to $87.124 \mathrm{deg}$ in $F M(p<0.001)$. At $t 3$, no significant differences between motion conditions were found. The mean phase margin was $126.335 \mathrm{deg}$ for $N M, 111.755$ for $G H, 106.012 \mathrm{deg}$ for $E H$ and $105.594 \mathrm{deg}$ for $F M$. At time segment $t 4$, there were also no significant differences between motion conditions. The mean phase margin was $94.386 \operatorname{deg}$ for $N M$, $101.241 \mathrm{deg}$ for $G H, 89.990 \mathrm{deg}$ for $E H$ and $102.345 \mathrm{deg}$ for $F M$. The simple main effect of time revealed that, in the $N M$ condition, the pitch phase margin did not significantly change from 83.407 deg at $t 1$ to 78.415 deg at $t 2$ 
( $p=1.000)$, but increased significantly from $83.407 \mathrm{deg}$ at $t 1$ to $127.349 \mathrm{deg}$ at $t 3(p<0.001)$ and to $95.286 \mathrm{deg}$ at $t 4(p=0.018)$. It also significantly increased from $78.415 \mathrm{deg}$ at $t 2$ to $127.349 \mathrm{deg}$ at $t 3(p<0.001)$, and to 95.286 deg at $t 4(p=0.003)$. Lastly, the pitch phase margin significantly decreased from $127.349 \mathrm{deg}$ at $t 3$ to $95.286 \mathrm{deg}$ at $t 4(p<0.001)$. For motion condition $G H$, the phase margin significantly decreased from 89.662 deg at $t 1$ to 78.707 deg at $t 2(p=0.008)$ and increased to $119.409 \mathrm{deg}$ at $t 3(p=0.002)$. It did not significantly change compared to the phase margin at $t 4(p=0.618)$. The pitch phase margin significantly increased from $78.707 \mathrm{deg}$ at $t 2$ to 119.409 deg at $t 3(p=<0.001)$ and to $98.167 \mathrm{deg}$ at $t 4(p=0.016)$. Lastly for the $G H$ condition, the pitch phase margin significantly decreased from $119.409 \mathrm{deg}$ at $t 3$ to $98.167 \mathrm{deg}$ at $t 4(p=0.007)$. In the $E H$ motion condition, the pitch phase margin did not significantly change between $79.621 \mathrm{deg}$ at $t 1$ and $70.732 \mathrm{deg}$ at $t 2(p=0.116)$ and $85.841 \mathrm{deg}$ at $t 4(p=0.519)$. It did increase from $79.621 \mathrm{deg}$ at $t 1$ to $107.979 \mathrm{deg}$ at $t 3(p=0.002)$. There was also a significant increase from $70.732 \mathrm{deg}$ at $t 2$ to $107.979 \mathrm{deg}$ at $t 3(p<0.001)$ and to $85.841 \mathrm{deg}$ at $t 4(p=0.041)$. The pitch phase margin significantly decreased from $107.979 \mathrm{deg}$ at $t 3$ to $85.841 \mathrm{deg}$ at $t 4(p=0.027)$. Finally, in the $F M$ condition, the phase margin did not significantly change between $90.402 \mathrm{deg}$ at $t 1$ and $88.245 \mathrm{deg}$ at $t 2(p=1.000)$, significantly increased to $119.295 \mathrm{deg}$ at $t 3(p=0.001)$ and significantly increased to $101.264 \mathrm{deg}$ at $t 4(p=0.010)$. It also significantly increased from $88.245 \mathrm{deg}$ at $t 2$ to $119.295 \mathrm{deg}$ at $t 3(p=0.001)$ and to $101.264 \mathrm{deg}$ at $t 4(p=0.044)$. Lastly for the $F M$ condition, pitch phase margin significantly decreased from 119.295 deg at $t 3$ to 101.264 deg at $t 4$ $(p=0.047)$.

\section{Discussion}

A stall recovery task was performed under different simulator motion conditions. Pilots had to follow a flight director that guided them through the approach to stall and stall recovery maneuvers. Disturbance forcing functions were added in order to identify pilot control behavior in both pitch and roll axes. The stall task was divided into four segments (Fig. 2). The first segment (S1) consisted of a level flight at $40000 \mathrm{ft}$. Then, pilots had to pitch up in order to enter a stall $(S 2)$, after which they entered a dive in order to increase their airspeed and recover from the stall $(S 3)$. In the last segment $(S 4)$, pilots had to pull up slowly to recover the airplane to the original level flight condition. Pilots flew the task under four motion conditions: no motion $(N M)$, generic hexapod motion $(G H)$, enhanced hexapod motion $(E H)$, and full motion $(F M)$.

Subjective motion ratings were collected in order to analyze how the motion in the no motion and hexapod motion conditions compared to that in the full motion condition. As expected, the condition with no motion was rated the lowest, followed by the generic hexapod condition and the enhanced hexapod condition. In general, the $E H$ and $F M$ motion conditions felt most similar. However, there was no statistically significant difference between how pilots rated the $G H$ and $E H$ conditions. In the last segment, $S 4$, the motion felt more violent than in $S 1$, especially for the $E H$ and $F M$ motion configurations. This might be due to the fact that the airspeed and air density were higher in $S 4$ compared to $S 1$.

Task performance was defined by the RMS of the error signal in both axes. Roll error RMS was the highest for the $N M$ condition, indicating worse performance, and the lowest for the $E H$ and $F M$ conditions, indicating better performance. Furthermore, the roll error RMS slightly increased from $t 1$ to $t 4$, most likely due to the more violent motion in $t 4$ compared to $t 1$. Similar trends were observed for the pitch axis. However, for the first three time segments, there was no significant difference in pitch performance between the $G H, E H$ and $F M$ conditions. In the last time segment the best performance was found for the $E H$ motion condition. Control activity was calculated from the RMS of the control input. It is interesting to note that for roll, the lowest control input was in the $N M$ condition, whereas there were no significant differences between the conditions with motion. In the pitch axis however, the highest control input was observed for the $F M$ condition, but no difference was observed for the other three conditions. This could mean that for roll, pilots controlled with more confidence whenever motion was available, whereas in pitch they controlled with more confidence in the full motion case only. This difference between roll and pitch cannot readily be explained.

Pilot model parameters were identified using a DEKF. For the roll position gain, no differences were found between motion conditions during the level flight segments $S 1$ and $S 4$, however in the approach to stall and dive maneuvers, the roll $K_{p}$ was the smallest for the $N M$ condition compared to the conditions in which motion was present. No significant differences were found between the flight segments. Roll velocity gain was significantly affected by motion conditions. It was the highest under $E H$ and lowest under $N M$, indicating that pilots increased their velocity gain with more motion. The reason why the roll velocity gain is highest for the $E H$ and not for the $F M$ condition might be due to increased false tilt motion cues in $E H$ compared to $F M$. The roll velocity gain increased at the end of $S 2$ compared to $S 1$, meaning that pilots acted more on velocity information close to stall, probably due to the decreased 
aircraft stability close to the stall point. The roll $K_{v}$ was smaller at the end of the $S 4$ segment compared to $S 1$, most likely as the aircraft still had not returned fully to the initial trim condition at the beginning of the flight. The neuromuscular frequency $\omega_{n}$ in roll did not change between motion conditions; however, it decreased going from $t 1$ to $t 2$ and $t 3$, and recovered back to the $t 1$ level in $t 4$. A lower roll neuromuscular frequency indicates that pilots act on less high-frequency disturbances during the approach to stall and the dive portion of the stall task. The pilot time delay was smaller in the $E H$ and $F M$ conditions compared to the $N M$ and $G H$ conditions by around $60 \mathrm{~ms}$. A possible explanation for this is that the increased motion made it easier for pilots to act quicker on the disturbances.

In the pitch axis, the pilot position gain $K_{p}$ showed a different behavior compared to roll. Motion did not introduce any significant change; however, at $t 3$ the pilot position gain significantly decreased, probably due to the large pitch attitude change at the end of the dive maneuver, which made pilots control more carefully in pitch in this time segment compared to the other time segments. The pitch velocity gain was the highest for the $F M$ condition, unlike the roll axis where the highest $K_{v}$ was obtained for the $E H$ condition. Unlike the roll neuromuscular frequency, the pitch neuromuscular frequency was significantly affected by the motion condition, which is an interesting find. The lowest pitch $\omega_{n}$ was obtained for the $E H$ condition, the next highest neuromuscular frequency was obtained for the $N M$ condition, then the $G H$ condition, and the highest pitch $\omega_{n}$ was found for the $F M$ condition. This order cannot be readily explained. Similar to the roll axis, the neuromuscular frequency in the pitch axis seems to decrease in the $S 2$ and $S 3$ segments compared to the $S 1$ and $S 4$ segments. The neuromuscular damping ratio in the pitch axis is the highest overall for the $G H$ condition, followed by the $F M$ condition and then the $N M$ and $E H$ motion conditions. These differences are the largest in $S 1$. At the end of the stall recovery task, neuromuscular damping is more similar for all motion conditions. The pilot time delay in the pitch axis is the lowest for the $E H$ condition. The highest time delay is found for the $N M$ task, an expected result. However, unlike the roll axis, the pilot time delay is similar under the $G H$ and $F M$ motion configurations.

The roll crossover frequency was higher for the conditions with motion compared to the no motion condition. It was the lowest at the end of $S 3$, most likely due to the slightly decreased stability of the aircraft as indicated by the lower aircraft dynamics break frequency in Fig. 13b. The roll phase margin was highest for the $E H$ condition and at $t 2$, as was expected since the aircraft roll dynamics were the most stable at $t 2$. In the pitch axis, the crossover frequency was the highest for the full-motion condition $F M$. The pitch crossover frequency was the lowest at the end of $S 3$, probably due to the large pitch attitude change, resulting in pilots not compensating for disturbances effectively, despite the fact that the pitch dynamics were the most unstable at the end of $S 2$ (Fig. 14b). It is also interesting to note that the largest difference between motion conditions occurred at $t 3$. Possibly indicating that the motion condition affects pitch open-loop performance the most where the aircraft response is more dynamic. Pitch phase margin was the lowest for the $E H$ condition and not the $F M$ condition. It was the highest at $t 3$, although the aircraft dynamics were more stable at $t 4$ (Fig. 14b). A possible explanation could be that the low pitch dynamics gain at $t 3$ resulted in an increase in open-loop phase margin.

The DEKF did provide accurate estimation results in both the roll and pitch axes. However, more accurate and consistent results were obtained in roll compared to pitch, confirming hypothesis H1. Especially the pilot pitch neuromuscular parameters showed behavior unlikely caused solely by the motion condition that cannot be readily explained.

Hypothesis $\mathrm{H} 2$ can be partially accepted, as the effects of motion on pilot control behavior and performance in roll were in line with previous research, but less so in pitch. The pilot position gain $K_{v}$ was the lowest in the $N M$ condition for both axes, and the highest in $E H$ for the roll axis and $F M$ for the pitch axis. Furthermore, the pilot position gain did not significantly change for the roll axis but did for the pitch axis. The differences in pilot velocity gain were much smaller between the $N M, G H$ and $E H$ conditions for the pitch axis compared to the roll axis. Finally, disturbance-rejection performance was more significantly affected by motion in roll compared to pitch. These findings provide useful insights into how different simulator motion settings affect pilot control behavior differently depending on the controlled axis. However, this obviously also depends on the type of task.

Pilot control behavior and performance significantly adapted as the aircraft dynamics changed throughout the stall task. Mainly the pilot equalization parameters changed over time. The pilot velocity gains increased closer to the stall point. Pilot position gains remained fairly constant between $t 1$ and $t 2$ but decreased in the stall recovery. This means $\mathrm{H} 3$ can be accepted. Finally, hypothesis $\mathrm{H} 4$ can be accepted as differences between motion configurations in most dependent variables increased closer to the stall point. A very clear example of this effect can be observed in the pilot roll position and velocity gains (comparing $t 1$ and $t 2$ ), and the disturbance-rejection performance in roll and pitch. 


\section{Conclusion}

A stall recovery task experiment was performed in the Vertical Motion Simulator at NASA Ames Research Center. Pilots had to follow a flight director into a stall and then into a recovery maneuver. Disturbances were added in both the pitch and roll axes in order to identify manual control parameters during different stages of the task using a novel time-varying parameter estimation method. Four simulator motion conditions were used: no motion, generic hexapod motion, enhanced hexapod motion, and full motion. Performance was highest for the enhanced hexapod and full motion conditions in both axes and the lowest for the condition with no motion. In the roll axis, the pilot position gain did not significantly change between time segments, but was the lowest for the condition with no motion. The pilot velocity gain was significantly different between motion conditions, the largest difference being found just before the stall point. The enhanced hexapod motion condition induced the highest pilot roll velocity gain. In the pitch axis, the pilot position gain was significantly different between time segments but not between motion conditions. The pilot pitch velocity gain was highest for the full motion condition and increased at the beginning of the stall, but did not change significantly for the other motion conditions. Neuromuscular frequency decreased for both roll and pitch axes at the beginning of the stall and in the recovery. There was no difference between motion conditions in the roll axis, unlike the pitch axis where significant differences in neuromuscular frequency were found. In summary, these findings suggest that the enhanced hexapod motion configuration induced pilot control behavior and performance more similar to that under the full motion configuration, but more so in roll compared to pitch. Furthermore, the different motion configurations affected control behavior and performance more strongly at different times during the stall maneuver; for example, close to the stall point. This might warrant the use of adaptive motion algorithms that emphasize different components of the total aircraft motion throughout a stall.

\section{Acknowledgements}

The authors thank everyone at NASA Ames SimLabs who contributed to the experiment. We especially thank Steven Norris and Emily Lewis for their daily support in setting up the experiment and operating the Vertical Motion Simulator. We thank the pilots who participated in the experiment. Finally, we would also like to thank Dr. Thomas Lombaerts for his help and support during the initial setup of the experiment. This work was supported by NASA's Technologies for Airplane State Awareness project for which Dr. Gautam Shah is the technical program coordinator.

\section{References}

${ }^{1}$ Federal Aviation Administration, Stall Prevention and Recovery Training, Nov. 2015, Advisory Circular 120-109A.

${ }^{2}$ Schroeder, J. A., "Research and Technology in Support of Upset Prevention and Recovery Training," Proceedings of the AIAA Modeling and Simulation Technologies Conference, Minneapolis (MN), No. AIAA-2012-4567, 13-16 Aug. 2012.

${ }^{3}$ McRuer, D. T. and Jex, H. R., "A review of quasi-linear pilot models," Human Factors in Electronics, IEEE Transactions on, , No. 3, 1967, pp. 231-249.

${ }^{4}$ Stapleford, R. L., Peters, R. A., and Alex, F. R., "Experiments and a Model for Pilot Dynamics with Visual and Motion Inputs," Tech. Rep. NASA CR-1325, NASA, 1969.

${ }^{5}$ Zaal, P. M. T., Pool, D. M., de Bruin, J., Mulder, M., and van Paassen, M. M., "Use of Pitch and Heave Motion Cues in a Pitch Control Task," Journal of Guidance, Control, and Dynamics, Vol. 32, No. 2, March-April 2009, pp. 366-377.

${ }^{6}$ Pool, D. M., Zaal, P. M. T., van Paassen, M. M., and Mulder, M., "Effects of Heave Washout Settings in Aircraft Pitch Disturbance Rejection," Journal of Guidance, Control, and Dynamics, Vol. 33, No. 1, Jan.-Feb. 2010, pp. $29-41$.

${ }^{7}$ Schroeder, J. A. and Grant, P. R., "Pilot Behavioral Observations in Motion Flight Simulation," Proceedings of the AIAA Guidance, Navigation, and Control Conference and Exhibit, Toronto (ON), Canada, No. AIAA-2010-8353, 2-5 Aug. 2010.

${ }^{8}$ Zaal, P., Popovici, A., and Zavala, M. A., "Effects of False Tilt Cues on the Training of Manual Roll Control Skills," AIAA Modeling and Simulation Technologies Conference, 2015, p. 0655.

${ }^{9}$ Klyde, D. H., Thompson, P. M., Bachelder, E. N., and Rosenthal, T. J., "Evaluation of Wavelet-Based Techniques for Detecting Loss of Control," Proceedings of the AIAA Atmospheric Flight Mechanics Conference and Exhibit, Providence (RI), No. AIAA-2004-4702, 16-19 Aug. 2004.

${ }^{10}$ Olivari, M., Nieuwenhuizen, F. M., Bülthoff, H. H., and Pollini, L., "Identifying Time-Varying Neuromuscular Response: Experimental Evaluation of a RLS-based Algorithm," Proceedings of the AIAA Modeling and Simulation Technologies Conference, Kissimmee, Florida FL, No. AIAA-2015-0658, 5-9 Jan. 2015.

${ }^{11}$ Zaal, P. M., "Manual Control Adaptation to Changing Vehicle Dynamics in Roll-Pitch Control Tasks," Journal of Guidance, Control, and Dynamics, 2016, pp. 1046-1058.

12 van Grootheest, A., Pool, D. M., van Paassen, M., and Mulder, M., "Identification of Time-Varying Manual Control Adaptations with Recursive ARX Models," 2018 AIAA Modeling and Simulation Technologies Conference, No. AIAA-2018-0118, American Institute of Aeronautics and Astronautics, jan 2018.

${ }^{13}$ Popovici, A., Zaal, P. M. T., and Pool, D. M., "Dual Extended Kalman Filter for the Identification of Time-Varying Human Manual Control 
Behavior," AIAA Modeling and Simulation Technologies Conference, No. AIAA-2017-3666, American Institute of Aeronautics and Astronautics, jun 2017.

${ }^{14}$ Hueschen, R. M., "Development of the Transport Class Model (TCM) Aircraft Simulation From a Sub-Scale Generic Transport Model (GTM) Simulation,” Technical Report NASA/TM-2011-217169, NASA Langley Research Center, Hampton, Virginia, 2011

${ }^{15}$ Hyde, D. C., Brown, F. R., Shweyk, K. M., and Shah, G. H., "Advanced Modeling and Uncertainty Quantification for Flight Dynamics (VSST1-7) Interim Results and Challenges," AIAA Atmospheric Flight Mechanics Conference, No. AIAA-2014-0035, American Institute of Aeronautics and Astronautics, jan 2014.

${ }^{16}$ Lombaerts, T., Schuet, S., Stepanyan, V., Kaneshige, J., Hardy, G., Shish, K. H., and Robinson, P., "Piloted Simulator Evaluation Results of Flight Physics Based Stall Recovery Guidance," 2018 AIAA Guidance, Navigation, and Control Conference, No. AIAA-2018-0383, American Institute of Aeronautics and Astronautics, jan 2018.

${ }^{17}$ Campbell, S., Kaneshige, J., Nguyen, N., and Krishnakumar, K., "An Adaptive Control Simulation Study using Pilot Handling Qualities Evaluations," AIAA Guidance, Navigation, and Control Conference, No. AIAA-2010-8013, American Institute of Aeronautics and Astronautics, aug 2010.

${ }^{18}$ Steurs, M., Mulder, M., and van Paassen, M. M., “A Cybernetic Approach to Assess Flight Simulator Fidelity," Proceedings of the AIAA Modelling and Simulation Technologies Conference and Exhibit, Providence (RI), No. AIAA-2004-5442, 16-19 Aug. 2004.

${ }^{19}$ McRuer, D. T., Graham, D., Krendel, E. S., and Reisener, W., "Human Pilot Dynamics in Compensatory Systems. Theory, Models and Experiments With Controlled Element and Forcing Function Variations," Tech. Rep. AFFDL-TR-65-15, Wright Patterson AFB (OH): Air Force Flight Dynamics Laboratory, 1965.

${ }^{20}$ Zaal, P. M. T. and Mobertz, X. R. I., "Effects of Motion Cues on the Training of Multi-Axis Manual Control Skills," AIAA Modeling and Simulation Technologies Conference, AIAA, Denver, Colorado, 2017.

${ }^{21}$ Beard, S. D., Reardon, S. E., Tobias, E. L., and Aponso, B. L., "Simulation System Optimization for Rotorcraft Research on the Vertical Motion Simulator," Proceedings of the AIAA Modeling and Simulation Technologies Conference, Minneapolis (MN), No. AIAA-2012-4634, 1316 Aug. 2012.

${ }^{22}$ International Civil Aviation Organization, ICAO 9625: Manual of Criteria for the Qualification of Flight Simulation Training Devices. Volume 1 - Aeroplanes, 2009, 3rd edition.

${ }^{23}$ Zaal, P. M. T., Pool, D. M., Chu, Q., Mulder, M., Van Paassen, M., and Mulder, J. A., "Modeling Human Multimodal Perception and Control using Genetic Maximum Likelihood Estimation," Journal of Guidance, Control, and Dynamics, Vol. 32, No. 4, 2009, pp. $1089-1099$.

${ }^{24}$ Shirley, R. S. and Young, L. R., "Motion Cues in Man-Vehicle Control," Fourth Annual NASA-University Conference on Manual Control, University of Michigan, Ann Arbor (MI), 21-23 March 1968, pp. 435-448. 